\title{
Correlation of SA349/2 Helicopter Flight Test Data with a Comprehensive Rotorcraft Model
}

Gloria K. Yamauchi, Ruth M. Heffernan, and Michel Gaubert

\author{
(NASA-TM-88351) CORBEIATICN CF SA349/2 \\ HELICCPTEB FLIGBT-TEST CATA WITE A \\ CCAPREHENSIVE RCTCECRAFT MCDEI (NASA) $56 \mathrm{p}$ \\ CSCL 010 Unclas \\ G3/05 43359
}

February 1987

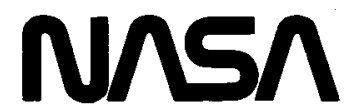

National Aeronautics and

Space Administration 


\section{Correlation of SA349/2 Helicopter Flight Test Data with a Comprehensive Rotorcraft Model}

Gloria K. Yamauchi,

Ruth M. Heffernan, Ames Research Center, Moffett Field, California Michel Gaubert, Aerospatiale-Division Helicopters, Marignane, France 
Note

This report is a modified version of the paper (same title) presented at the Twelfth European Rotorcraft Forum, September 22-25, 1986, Garmisch-Partenkirchen, Federal Republic of Germany. Errors in the blade section radius of gyration distribution used by the analysis have been corrected. Also, corrections to the measured pressure data have been made. 


\title{
CORRELATION OF SA349/2 HELICOPTER FLIGHT-TEST DATA \\ WITH A COMPREHENSIVE ROTORCRAFT MODEL
}

\section{Gloria K. Yamauchi and Ruth M. Heffernan NASA Ames Research Center Moffett Field, California, U.S.A.}

and

\author{
Michel Gaubert \\ Aerospatiale - Division Helicopteres \\ Marignane, France
}

\section{Abstract}

A comprehensive rotorcraft analysis model was used to predict blade aerodynamic and structural loads for comparison with flight test data. The data were obtained from an SA349/2 helicopter with an advanced geometry rotor. Sensitivity of the correlation to wake geometry, blade dynamics, and blade aerodynamic effects was investigated. Blade chordwise pressure coefficients were predicted for the blade transonic regimes using the model coupled with two finite-difference codes.

\section{SYMBOLS}

c.g. center of gravity

$C_{\ell} \quad$ blade section $l$ ift coefficient

$c_{n} \quad$ blade section normal force coefficient

$C_{p} \quad$ blade chordwise surface pressure coefficient

$\mathrm{C}_{\mathrm{T}} / \sigma$ ratio of rotor thrust coefficient to solidity

$r \quad$ blade radial station

$\mathrm{R}$ rotor radius

$r_{c} / c$ ratio of tip vortex core size to blade chord

a blade angle of attack

$\dot{\alpha} \quad$ time derivative of blade angle of attack

$\mu \quad$ advance ratio

$\psi \quad$ rotor blade azimuth station 
A joint rotorcraft research program has been established between the National Aeronautics and Space Administration (NASA) and the French Ministry of Defense. The purpose of the program is to correlate the NASA prediction code Comprehensive Analytical Model of Rotorcraft Aerodynamics and Dynamics (CAMRAD) with flight test data from the French SA349/2 Helicopter and an advanced geometry rotor system.

Structural and aerodynamic loads and performance were measured for several thrust levels and advance ratios. From the flight envelope, 16 flight conditions were selected by NASA and Aerospatiale for documentation. In addition, data from three of these flight conditions were chosen for detailed correlation with CAMRAD. These conditions provided an opportunity to exercise various modeling features within CAMRAD.

The pressure data from this flight test are a valuable addition to the limited data base of helicopter rotor blade pressures in forward flight, especially in the transonic regime. Predictions of shock formations near blade tips using CAMRAD coupled with a finite-difference code have been restricted to comparisons with several model rotor data sets using rigid blades (described in Ref. 1). Chordwise pressure comparisons presented here aid in further validating the coupling procedure.

The flight test, the comprehensive model, the assumptions and approximations in modeling the aircraft and rotor in CAMRAD, and the resulting theoretical predictions compared with the data are described in this paper. Finally, recommendations for improving the correlation are given.

\section{AIRCRAFT DESCRIPTION}

The flight tests were performed on an Aerospatiale Gazelle SA349/2 helicopter (Fig. 1) dedicated to experimental research. The SA349/2 belongs to the Gazelle family of helicopters, but differs somewhat from the 342 line of aircraft. The SA349/2 includes a comprehensive on-board data acquisition system. Table 1 provides general characteristics of the helicopter. For this flight test program, the aircraft was fitted with the Aerospatiale advanced geometry rotor. The rotor includes the classical articulated Non Articule en Trainee (NAT) hub (Fig. 2) and three research Grande Vitesse (GV) blades. The NAT hub, which is the standard Gazelle hub, is fully articulated and has a lag damper with nonlinear characteristics (stiffness and damping).

The GV blades were developed to achieve a better understanding of rotor aerodynamics and dynamics. Features of the blades are (Fig. 3) OA209 advanced airfoils ( $9 \%$ thickness), a removable tip, and an adjustable blade section c.g. location. For this flight test, a rectangular tip and a blade c.g. location of $24.7 \%$ chord were used. The OA209 airfoil is discussed in Ref. 2. The twist distribution (Eig. 3b) is 
constant at the tip, linear on the main part of the blade, and varies 11.5 degrees from the root to the first airfoiled section.

The tail rotor for the $\mathrm{SA} 349 / 2$ is a Fenestron. The Fenestron is a shrouded 13-bladed rotor with one control input (collective pitch control).

Detailed technical data on the aircraft and rotors are available from Ref. 3 .

\section{ELIGHT TEST INSTRUMENTATION}

The rotating frame instrumentation included both blade pressure transducers and strain gages. Strain gages for measuring pitch-link loads and flap and lag angle were also available.

The blade pressure data analyzed in this paper were obtained from 51 pressure transducers distributed between two blades, as shown in Fig. 4. Three groups of 20 chordwise transducers (upper and lower surface) were located at $75 \%, 88 \%$, and $97 \%$ radial stations. The number of functional transducers were 18,15 , and 18 for $75 \%, 88 \%$, and $97 \%$ radial station, respectively.

The blade structural data in this paper were obtained from 15 strain gages, also shown in Fig. 4. The gages (four flap-bending, seven lag-bending, and four torsion gages) are located at eight radial stations on two blades. Data from additional blade strain gage instrumentation installed on these two blades were not recorded because of slipring limitations.

Instrumentation in the fixed frame included strain gages on the primary rotor control system servos and main gear box struts. Pilot and copilot seat vertical acceleration were also measured. The following flight condition parameters were recorded: ground pressure and temperature, altitude, velocity, static temperature (in flight), static pressure (in flight), collective pitch stick position, lateral and longitudinal cyclic pitch stick position, load factor, rotor speed, sideslip angle, aircraft pitch, roll and yaw speed, engine power and fuel consumption.

\section{DATA REDUCTION}

A total of 18 consecutive rotor revolutions were required to obtain all of the blade pressure data. Data from each of the three groups of 20 pressure transducers were acquired as time-histories over a period of six consecutive rotor revolutions (starting from 0 degrees azimuth). The pressure signals were digitized at a frequency of 180 samples/rotor revolution. The data were then converted into physical units and transformed into aerodynamic coefficients. The strain gage signals were obtained as harmonic analysis coefficients ( 10 harmonics) calculated for one rotor revolution. The blade strain gage data 
were acquired during one of the 18 revolutions of pressure data acquisition. The blade strain gage data were next transformed into bladebending and torsional moments after accounting for the blade weight contribution and flap/lag/torsion interaction components. Figure 5 illustrates the data reduction procedure.

\section{FLIGHT CONDITIONS}

Various operating conditions were included in the orignal flight test envelope: (1) hover out-of-ground-effect; (2) level flights at $305 \mathrm{~m}, 1524 \mathrm{~m}$, and $3048 \mathrm{~m}(1000 \mathrm{ft}, 5000 \mathrm{ft}$, and $10000 \mathrm{ft}$ ) with speeds from $100 \mathrm{~km} / \mathrm{hr}$ to $300 \mathrm{~km} / \mathrm{hr}$; (3) steady turns at $305 \mathrm{~m}, 1524 \mathrm{~m}$, and $3048 \mathrm{~m}$ ( $1000 \mathrm{ft}, 5000 \mathrm{ft}$, and $10000 \mathrm{ft}$ ) corresponding to a range of load factor from 1.4 to 2.0 ; (4) autorotation at $200 \mathrm{~km} / \mathrm{hr}$; and (5) high speed dive at $350 \mathrm{~km} / \mathrm{hr}$.

From this flight envelope, 16 flight points were chosen for documentation purposes. The selected points provide a large range of advance ratios $(0.13<\mu<0.36)$ and thrust-to-solidity ratios $\left(0.062<\mathrm{C}_{\mathrm{T}} / \sigma<0.133\right)$, as shown in $\mathrm{Fig} .6$. The data are documented in Ref. 3; in addition, further details of the flight test instrumentation and data reduction process are explained in Ref. 3.

\section{DESCRIPTION OF THE COMPREHENSIVE ROTORCRAFT MODEL}

CAMRAD is an analysis designed to calculate rotor performance, aerodynamic and structural loads, aircraft vibration and gust response, flight dynamics and handling qualities, and aeroelastic stability. The analysis development is discussed in Refs. 4-6. Summaries of the various structural and aerodynamic models used in CAMRAD are described in Ref. 7. A general two-rotor aircraft with either articulated, hingeless, gimballed, or teetering rotors can be modeled using CAMRAD. The rotor structural model is based on engineering beam theory for rotating wings with large pitch and pretwist. The rotor aerodynamic model is based on lifting-line theory, and uses two-dimensional airfoil characteristics and a vortex wake. Three levels of aerodynamic analys is exist in CAMRAD: uniform inflow (linear variation of inflow over the rotor disk), nonuniform inflow with prescribed-wake geometry, and nonuniform inflow with a free-wake geometry. Figure 7 outlines the aircraft trim solution procedure in CAMRAD.

\section{APPROACH}

The strategy for the correlation effort was to first develop a baseline model of the SA349/2 aircraft and rotor for CAMRAD. Much time and effort was spent obtaining detailed information about the GV blades, NAT hub, and aircraft geometry. Also, definitions, sign conventions, and reference systems for the data and theory were constantly checked for consistency. 
Preliminary comparisons of theory with data were then performed. CAMRAD predictions were performed for seven flight conditions; the flight parameters for these conditions are shown in Table 2. Detailed results for the first three of the seven conditions will be presented here. These three flight points represent forward flight extremes: low speed, low thrust; high speed, low thrust; and high speed, high thrust. As a result of the preliminary CAMRAD calculations, several approximations and assumptions about both theory and data were necessary. The results presented in this paper are qualified by the following:

1. Measured mean values of blade loads proved to be unrealistic; therefore, all strain gage time-history data presented in this paper have the mean value removed.

2. Measured blade flap and lag angles are not presented since their accuracy is suspect, especially at high forward speed.

3. Measured lift coefficient is actually the normal force coefficient $C_{n}$ (the force perpendicular to the chord line). At small blade angle of attack, the difference between $C_{\ell}$ and $C_{n}$ is insignificant. For flight conditions where the blade experiences large angles of incidence, the error that is caused by the difference between $C_{\ell}$ and $C_{n}$ will be a small part of the overall error in predicting $C_{\ell}$. Large angles of incidence occur when the blade experiences dynamic stall, and as will be shown, this phenomenon is not well-predicted.

4. Measured blade moment coefficient values are unreliable, probably because of an insufficient number of chordwise pressure measurements.

5. The steadiness of Condition 3 data is questionable. Variations in the flight parameters were noticed during a period of 18 rotor revolutions of static data acquisition.

6. Fuselage aerodynamic characteristics used in CAMRAD were obtained from a wind tunnel test of a one-seventh-scale model of the SA349/2 fuselage.

7. The same aircraft c.g. location was used for all flight conditions.

8. CAMRAD normally calculates the blade-bending loads at a given radial station, $r$, by computing the difference between the total aerodynamic and inertial moments on the blade surface outboard of $r$. Using this method for the advanced-geometry rotor, the flapwise bending moments were found to be very sensitive to the blade mass distribution. This sensitivity is numerical, not physical. The blade-bending moments were, therefore, calculated directly from the blade curvature and stiffness. This method significantly improved the correlation of the blade loads; however, this method is not accurate where discontinuities in the blade stiffness distribution occur because of the modal 
structural model in CAMRAD. For the GV blades, jumps in the flapwise and edgewise stiffness occur near the 10\% and go\% radial stations. Poor correlation for bending moments near these radial stations is therefore expected.

9. The measured control inputs were used as initial control settings in the free-flight trim analysis in CAMRAD. In the trim iteration, the control settings were adjusted until force and moment equilibrium on the aircraft were achieved. For Condition 3, however, the aircraft was also trimmed to the measured power (descent). When trimming Condition 3 to force and moment equilibrium alone (level flight), the trimmed power level was unrealistically high.

Baseline modeling conditions were next developed for the seven flight conditions. Table 3 provides baseline values used in CAMRAD. Modeling parameters in CAMRAD were then varied to assess the sensitivity on the correlation; these parameters included: number of blade-bending and torsion modes; wake geometry; tip-vortex core size; lifting-surface correction; and blade stall model.

8. RESULTS

\section{Prediction of Control Inputs and Power Required}

Figure $8 \mathrm{a}$ presents predicted collective pitch as a function of advance ratio. Except for the turning flight conditions (Conditions 3 and 7 ), the collective is overpredicted by approximately 0.5 degrees for all conditions. The nearly constant of set in collective results from a tension-torsion coupling caused by the jump in blade twist at the inboard radial station (Fig. 3). This steep jump in twist was discovered to be the source of a steady, nose-down blade pitch. CAMRAD calculates a pitch increment from the tension-torsion coupling associated with the pretwist. A small positive pitch increment results from the negative twist inherent over most of the GV blade, and a large negative pitch increment results from the large positive pitch change at the root. The net elastic pitch change is negative and occurs at the blade root. The pitch increment is also steady because the change is produced by the centrifugal force. Since the analysis trims the helicopter to equilibrium flight, the primary effect is on the main rotor collective pitch.

CAMRAD models this tension-torsion coupling similar to Ref. 8 . Reference 9 presents a more general theory to account for this effect. Because of lack of mean torsion moment and pitch link load data, the predicted elastic pitch increment could not be checked directly. Figure $8 \mathrm{a}$ indicates that the magnitude of the elastic pitch increment is slightly overestimated. Calculations incorporating the general theory of Ref. 9 were performed by introducing an effective blade section radius of gyration parameter into CAMRAD. These calculations are also shown in Fig. 8a. The overall effect of implementing the general theory is to reduce the influence of the blade pretwist in the calculation of 
the collective pitch. The magnitude of the influence depends on the blade shear modulus and the useful load-bearing, cross-sectional area of the blade, which are used to compute the effective radius of gyration parameter. Because the GV blade is a composite structure, the approximation used for the shear modulus was very rough.

Figure $8 \mathrm{~b}$ and $8 \mathrm{c}$ present lateral and longitudinal cyclic pitch, respectively, as a function of advance ratio. The lateral cyclic pitch is overpredicted by an average of less than 0.5 degrees. The calculated longitudinal pitch is predicted to within 2 degrees (excluding Condition 7).

The prediction of total power required is shown in Fig. 9a. Condition 3 is not shown since measured power was used to trim the aircraft. Except for Condition 7, predictions for the remaining conditions are within an average of $5 \%$ of measured values. Aircraft attitude is not well-predicted for most of the flight conditions (Fig. 9b), probably because of an inaccurate representation of the fuselage aerodynamics.

8.2 Condition 1: low speed, low thrust $\left(\mu-0.14, C_{T} / \sigma=0.065\right)$

At low forward speed, the rotor wake tends to remain in the vicinity of the blades and strongly influences the blade aerodynamic environment. A detailed wake geometry is needed therefore for good aerodynamic load correlation.

Figure 10 presents $C_{\ell}$ as a function of azimuth for $r / R=0.75$, 0.88 , and 0.97 . Uniform inflow is not able to predict the details of the lift variation. The prescribed wake results are better, but still do not predict the details of the distribution. The free-wake geometry, however, is able to predict the trend and magnitude of the lift. At this low speed, the rotor wake is slowly convected away from the rotor allowing time for interaction between the blade and shed tip-vortices. This interaction is evident in Fig. 10 for $r / R=0.97$ on the retreating side of the rotor disk (note sharp peaks in $C_{\ell}$ near 270 degrees azimuth). Figure 11 shows the effect of varying tip-vortex core size on $C_{l}$. A ratio of core size to blade chord of 0.525 was found to be optimum. The core size used in CAMRAD includes other effects of blade/vortex interaction which are not modeled by the theory; therefore, the core size in CAMRAD is larger than the physical tipvortex core size.

For cases where the blade passes close to a tip-vortex (as in Condition 1), lifting-line theory usually cannot predict blade loads accurately, especially at the tip. A lifting-surface solution is required for these cases. CAMRAD uses a correction based on a linear, lifting-surface theory to adjust the solution for the vortex-induced loading on the blade which is calculated by lifting-line theory. Because of computation time constraints, this correction is only applied when the blade and vortex are a specified distance apart. Figure 12 
shows $C_{\ell}$ predictions using the lifting-surface theory correction. when usiñ̄g this eôriectioñ, a more realistic tip-vortex core size is allowed $\left(r_{c} / c=0.10\right.$ was used for this figure). For this flight condition, the calculated correction to lifting-line theory is small. The effect of tip-vortex core size is greater than the effect of the lifting-surface correction on $C_{\ell}$.

The flapwise bending moment is well-predicted at the $54 \%$ radial station, but not at the $20 \%$ and $80 \%$ stations (Fig. 13). Although the free-wake geometry results are a significant improvement over the uniform inflow results, the free wake provides only a small improvement in the predictions as compared to the prescribed wake results. The edgewise bending moment is predicted both in magnitude and trend for $r / R=0.20$, predicted in trend for $r / R=0.54$, and not well-predicted for $r / R=0.80$ ( $F i g .14)$. The difference between wake geometries becomes more significant near the blade outboard regions. The torsional moments are not well-predicted for any radial station, as shown in Fig. 15. If the one-half peak-to-peak values for data and theory were compared, however, the correlation would be quite reasonable. The data show a $5 / \mathrm{rev}-6 / \mathrm{rev}$ influence that is not accounted for by the theory. CAMRAD calculates the first torsion frequency to be $3.9 / \mathrm{rev}$.

The predicted pitch-link loads show a large $1 / \mathrm{rev}$ influence for both uniform and nonuniform inflow, whereas the data do not (Fig. 16). A possible explanation is the value used for the control system stiffness in CAMRAD (a very large value was used). A better estimation of the stiffness may improve the correlation.

8.3 Condition 2: high speed, low thrust $\left(\mu=0.36, C_{T} / \sigma=0.071\right)$

At high forward speed, the rotor wake is rapidly convected downstream, and so wake effects on blade loading are smaller. Figure 17 shows that the nonuniform inflow predictions of lift are slightly better than are the uniform inflow predictions. However, the difference between the free-wake and the prescribed-wake geometry results are insignificant. Henceforth, predictions for this flight condition are based on nonuniform inflow with a prescribed wake geometry.

The flapwise bending moments are reasonably well-predicted; better correlation is achieved at the outboard radial stations than at the inboard radial stations (Fig. 18). Using six blade-bending modes provides better predictions at the outboard radial stations than using five blade modes. The difference between using five and six blade modes is noticeable; however, there is no difference in results from using six, seven, or eight bending modes. The seventh and eighth bending modes are dominated by edgewise motion; therefore the flapwise moment predictions are not affected by these modes. The edgewise moments are not well-predicted on the advancing side of the rotor disk (Fig. 19), probably reflecting compressibility effects for this high-speed case. For the retreating side of the disk, the moments are better predicted at the inboard radial stations. Using five or six blade modes appears to 
provide the closest correlation. Figure 20 shows that pitch-link loads are badly predicted. Varying the number of blade modes does not improve the correlation.

Reasonable correlation is obtained for torsional moments at $20 \%$ and $54 \%$ radial stations ( $\mathrm{Fig} .21$ ). Increasing the number of torsion modes from two to four does not improve the predictions. The $5 / \mathrm{rev}-$ $6 / \mathrm{rev}$ influence present in Condition 1 is absent here.

\subsection{CAMRAD/Finite-difference predictions of surface pressures}

With an advancing tip Mach number of 0.87 , chordwise surface pressure data for this flight condition reveal shocks forming near the blade tip. Reference 10 describes a method for predicting transonic flows on rotors in forward flight. The predictions are made via a loose coupling ( $f$ ile transfer) between CAMRAD and a small-disturbance, finitedifference code (FDR). Recently, comparisons were made of results from FDR and several other finite-difference codes coupled with CAMRAD (Ref. 1). Results obtained by using one of these codes, a fullpotential rotor code (FPR), in addition to results from FDR, will be presented here. FPR is described fully in Ref. 11. The code solves a three-dimensional, conservative, full-potential equation to calculate unsteady rotor flows.

As explained in Ref. 10, CAMRAD first computes the blade partial angle of attack for the advancing side of the disk. The finitedifference code then computes the blade lift (for $r / R \geq 0.5$ ) for the advancing side of the disk. This is defined here as the end of the first iteration. The $C_{\ell}$ 's computed by the finite-difference code are then used by CAMRAD to compute new blade angles, which are used by the finite-difference code to compute a new $C_{\ell}$ distribution (end of second iteration). The iteration continues until the difference between the $\mathrm{C}_{\ell}$ calculated by CAMRAD and the finite-difference code becomes small.

Results from Ref. 1 are for rigid blade motion. As shown in Table 3, six bending modes and two torsion modes were used for the calculations presented here in Figs. 22 to 25.

Figures 22 and 23 present lift coefficient predictions for the advancing side of the rotor disk for FPR and FDR, respectively, for Condition 2. As stated earlier, blade lift information is passed from the finite-difference code to CAMRAD for the advancing side of the disk only. For the retreating side of the disk, lift information from the previous CAMRAD iteration is used. The step change in lift at 0 and 180 degrees azimuth can excite unwanted blade motion, especially for an elastic blade. In Fig. 22 and 23, the second iteration predictions are slightly oscillatory in nature. To avoid this problem, the step change in lift at 0 and 180 degrees azimuth should be made small. Ideally, inflow and lift information for the entire rotor disk should be exchanged between CAMRAD and the finite-difference code. 
Figures 22 and 23 show that using the coupling procedure between CAMRAD and either finite-difference code does not improve the prediction of $\mathrm{Cl}$ (for this flight condition). In fact, the lift is underpredicted at all locations, except in the region of $\psi=120$ to 160 degrees azimuth for FPR and in the region $\psi=100$ to 140 degrees azimuth for FDR. In these two regions, the second iteration results overpredict the lift. Figure 23 shows that FDR significantly underpredicts the lift near 0 degrees and 180 degrees azimuth for all three radial stations. In general, lifting-line theory does an adequate job of predicting the section lift. The reason for underpredicting the lift in Figs. 22-23 with both finite-difference codes is difficult to determine without a tip-path-plane angle-of-attack measurement.

Figure 24 and 25 show chordwise surface pressure coefficient (Cp) predictions for the three radial stations at three azimuth locations for FPR and FDR, respectively. The pressure predictions are again for Condition 2. As shown in Fig. 24, the shape of the $\mathrm{Cp}$ distribution predicted by FPR compares well with the data for all locations. The upper surface pressure peak is consistently underpredicted (because the calculated lift is too low). There is little difference between the first and second iteration results except at $\psi=150$ degrees (reflecting the trend in lift shown in Fig. 22). At $\psi=150$ degrees, the upper surface pressure peak is closely matched using the second iteration results (although the lower peak is better predicted using the first iteration results). Figure 25 shows similar results for the FDR predictions. The shape and magnitude of the pressure distributions are not as well predicted as compared to the FPR results. In particular, FPR predicts the lower surface pressure peak better than FDR. As in Fig. 24, differences between the first and second iteration results are small. FPR reasonably predicts the shock locations for $r / R=0.88$ and 0.97 at $\psi=150$ degrees (Fig. 24 b) and c)). FDR, however, does not predict these shock locations (Fig. 25 b) and c)).

8.5 Condition 3: high speed, high thrust $\left(\mu=0.34, C_{T} / \sigma=0.125\right)$

Although not shown here, there is little difference between the prescribed- and free-wake results for this condition. Since the prescribed-wake analysis is computationally less expensive than the free-wake analysis, calculations were performed using the prescribedwake geometry. The high thrust level of this flight condition leads to stall on the retreating side of the rotor disk (especially near the blade inboard region). The rapid blade pitch motion causes a delay in the occurrence of separated flow. Once the blade has reached stall, a vortex is shed from the leading edge resulting in large transient blade loads. Therefore, a dynamic stall model is used in the calculations for this flight condition. Several models of dynamic stall are available in CAMRAD. In the model used here (based on Refs. 12-14), a correction that is proportional to $\dot{\alpha}$ is applied to $a$ before the blade section lift, the drag, and the moment coefficients are evaluated. This model accounts for the hysteresis of dynamic stall, but not for the leadingedge vortex effects. 
For the $75 \%$ radial station, Fig. 26 shows that the static stall model is not able to predict the complex $C_{\ell}$ behavior on the retreating side of the rotor disk. The dynamic stall model provides a better prediction in this region of the disk. The difference between the two stall model predictions on the retreating side of the disk becomes less significant for the outer radial stations since the blade becomes less stalled near the blade tip. As an additional comparison, one revolution of unaveraged data is included. Attempts were made to improve the predictions by varying the correction term to the blade angle of attack; however, significant improvements were not obtained. A more sophisticated stall model will be necessary to predict the detailed behavior of $\mathrm{C}_{\ell}$.

Figure 27 shows that the flapwise moments are reasonably wellpredicted. No significant improvement is gained by using the dynamic stall model instead of the static stall model. On the other hand, the edgewise moment at $20 \%$ radial station is better predicted by the dynamic stall model on the retreating side of the disk (Fig. 28a), where the blade is most stalled. The edgewise moments at the outer radial stations are not well-predicted by either stall model. For all three radial stations shown in Fig. 29 , the torsional moments are not wellpredicted on the retreating side of the disk by either model. Figure 30 again shows the poor correlation of pitch-link loads. Using the dynamic stall model does not improve the correlation.

\section{RECOMMENDATIONS FOR CORRELATION IMPROVEMENT}

1. Since the one-seventh-scale data does not represent the fullscale fuselage aerodynamic characteristics exactly, additional calculations were performed to assess the sensitivity of the correlation to fuselage drag. For Condition 2, changing the fuselage drag had a pronounced effect on the trimmed control inputs, power required, and blade loads. The predictions were also somewhat sensitive to the fuselage moment characteristics. For future correlations with this data set, using full-scale fuselage aerodynamic characteristics would improve the accuracy of the predictions.

2. Since the blade flap angle measurements were suspect, the rotor position relative to the fuselage was unknown. If CAMRAD were trimmed to the measured tip-path-plane angle, the resulting predictions could improve.

3. Incorporating a more sophisticated dynamic stall model into CAMRAD may improve the predictions of $C_{\ell}$ for stalled regions of the blade.

\section{CONCLUSIONS}

A summary of the correlation results for aircraft performance and trim and the three flight conditions are listed below. 
1. CAMRAD is able to predict aircraft performance to within an average of $5 \%$ for the level flight (low thrust) cases; performance for the turning flight (high thrust) cases is not well-predicted.

2. The collective pitch is consistently overpredicted by about 0.5 degrees. This overprediction is caused by the steep jump in twist at the blade inboard section which produces a steady, nose-down blade pitch increment. Introducing an effective blade section radius of gyration parameter reduces the effect of the pretwist on the calculated collective.

3. Lateral cyclic pitch is overpredicted by roughly 0.5 degrees and longitudinal cyclic pitch is predicted to within 2 degrees (both excluding Condition 7). Aircraft attitude is not well-predicted.

10.2 Condition $1\left(\mu=0.14, C_{\mathrm{T}} / \sigma=0.065\right)$

1. Blade tip-vortex interactions dominate the wake effects on blade loads. Tip-vortex core size has pronounced effects on the $C_{\ell}$ predictions.

2. The free-wake geometry is able to model the blade tip-vortex interactions.

3. Use of a lifting-surface theory correction has little effect on the $C_{\ell}$ prediction.

4. Nonuniform inflow is required to predict blade structural loads. The free wake is not important in predicting flapwise moments, but is required for calculating edgewise bending moments at outer radial stations. Torsional moments and pitch-link loads are not wellpredicted.

10.3 Condition $2\left(\mu=0.36, C_{T} / \sigma=0.071\right)$

1. Nonuniform inflow is required for reasonable predictions of blade aerodynamic and structural loads; however, there is little or no difference between the free- and prescribed-wake results.

2. Use of five or six blade-bending modes provide the best predictions of bending moments. Flapwise moments were well-predicted for all radial stations. Edgewise moments are not well-predicted on the advancing side of the rotor disk; better correlation is achieved on the retreating side of the disk at inboard stations.

3. Variation of the number of blade-bending modes had no effect on the poor prediction of pitch-link loads.

4. Torsional moments were reasonably predicted at the inboard radial station. Increasing the number of torsional modes had a small effect on the predictions. 
5. Coupling CAMRAD with either FPR or FDR did not provide a better prediction of blade lift as compared to lifting-line theory predictions.

6. In general, the FPR code provided better predictions of blade surface pressures than the FDR code. The shape of the pressure distributions were well-predicted by FPR. However, both FPR and FDR underpredicted the magnitude of the upper surface pressures.

7. Shock locations were reasonably well-predicted by FPR.

10.4 Condition 3( $\left.\mu=0.34, \mathrm{C}_{\mathrm{T}} / \sigma=0.125\right)$

1. The difference between prescribed-wake and free-wake results is small.

2. A dynamic stall model provides a better prediction of $C_{\ell}$ on the retreating side of the rotor disk for the blade inboard sections than a static stall model. The detailed behavior of $C_{\ell}$, however, is not well-predicted by using the dynamic stall model.

3. The flapwise moments are reasonably well-predicted, although the dynamic stall model does not offer significant improvements over the static model results. The edgewise moments at the inboard radial stations are more sensitive to the stall model used; the edgewise moments at the outboard radial stations are not well-predicted.

4. Torsional moments on the retreating side of the disk and pitch-link loads are not well-predicted by either stall model.

\section{VALUE OF PERFORMING CORRELATION STUDIES}

The benefits of performing extensive correlations, such as presented here, can be assessed in several ways.

1. The theoretical model is checked for validity over a wide range of flight conditions.

2. Limitations of the analysis are identified.

3. The data set is validated somewhat by the analysis. Data reliability can be verified by rechecking instrumentation, data acquisition systems, and data reduction processes; however, gross errors in the data are easily identified by performing correlations with analyses. Errors made consistently throughout the data reduction process can also be identified by thorough correlation with a validated analysis. 
The authors would like to thank Dr. Wayne Johnson for his invaluable guidance throughout this joint project. We would also like to thank Dr. Chee Tung of the U.S. Army Aeroflightdynamics Directorate for his assistance with the finite-difference codes and useful discussions. Finally, we would like to thank Dr. William Warmbrodt of NASA Ames and Mr. Olivier Lambert of the French Ministry of Defense who are the technical representatives for this project; their continued efforts have made this project possible.

\section{REFERENCES}

1. F. Caradonna, C. Tung

A Review of Current Finite Difference Rotor Flow Methods.

Forty-Second Annual National Forum of the American Helicopter Society. Washington, D.C. (June 1986)

2. J. J. Thibert

A New Airfoil Family for Rotor Blades. European Rotorcraft and Powered Lift Forum. Aix-en-Provence, France (September 1977)

3. R. Heffernan, M. Gaubert Structural and Aerodynamic Loads and Performance Measurements of an SA349/2 Helicopter with an Advanced Geometry Rotor. NASA TM-88370 (November 1986)

4. W. Johnson

A Comprehensive Analytical Model of Rotorcraft Aerodynamics and Dynamics, Part I: Analysis Development. NASA TM-81182 (June 1980)

5. W. Johnson

Development of a Comprehensive Analys is for Rotorcraft - I, Rotor Model and Wake Analysis.

Vertica (1981) 5 (2)

6. W. Johnson

Development of a Comprehensive Analysis for Rotorcraft - II, Aircraft Model, Solution Procedure and Applications. Vertica (1981) 5(3)

7. W. Johnson Assessment of Aerodynamic Models in a Comprehensive Analysis for Rotorcraft. NASA TM-86835 (October 1985) 

bined Flapwise Bending, Chordwise Bending, and Torsion of Twisted Nonuniform Rotor Blades.

NACA Report 1346 (1958)

9. D. Hodges

Torsion of Pretwisted Beams Due to Axial Loading .

J. Appl. Mech. (1980) 47 (2). See also

Discussion, J. Appl. Mech. (1981) 48 (3)

10. C. Tung, F. X. Caradonna, The Prediction of Transonic Flows on

D. A. Boxwell,

W. Johnson

11. R. Strawn, F. Caradonna
Advancing Rotors.

Fortieth Annual National Forum of the American Helicopter Society. Arlington, VA (May 1984)

Numerical Modeling of Rotor Flows with a Conservative Form of the Full-Potential Equations.

AIAA 24 th Aerospace Sciences Meeting, Reno, Nevada, January 6-9, 1986, AIAA Paper No. 86-0079.

12. F. Harris, F. Tarzanin, R. Fisher

13. F. Tarzanin

14. R. Gormont
Rotor High Speed Performance, Theory vs. Test.

J. American Helicopter Soc. (1970) 15 (3)

Prediction of Control Loads due to Blade Stall.

J. American Helicopter Soc. (1972) 17 (2)

A Mathematical Model of Unsteady Aerodynamics and Radial Flow for Application to Helicopter Rotors. USAAVLABS TR 72-67 (May 1973) 
TABLE 1. SA349/2 AIRCRAFT CHARACTERISTICS

\begin{tabular}{lr} 
Aircraft gross weight & $2000 \mathrm{~kg}$ \\
Main rotor & 3 \\
number of blades & $5.25 \mathrm{~m}$ \\
radius & $0.35 \mathrm{~m}$ \\
blade chord & 0.0637 \\
solidity & $387 \mathrm{rpm}$ \\
rotational speed & $5919 \mathrm{rpm}$ \\
Tail rotor rotational speed & \\
Engine type & Astazou XIV H rated at $550 \mathrm{kw}$ \\
\hline
\end{tabular}




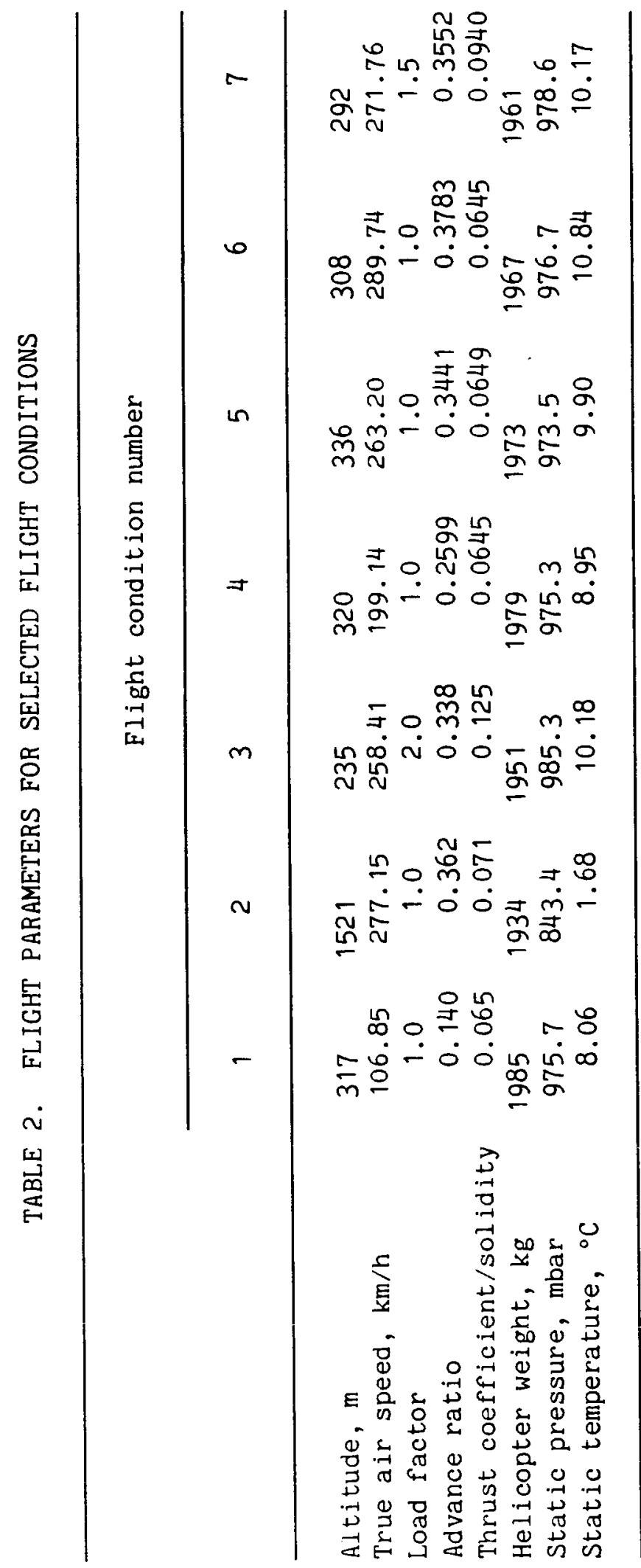




\begin{tabular}{cccccc}
\hline Condition & $\begin{array}{c}\text { Tip vortex } \\
\text { core size, } \\
r_{c} / c\end{array}$ & $\begin{array}{c}\text { Number of } \\
\text { bending } \\
\text { modes }\end{array}$ & $\begin{array}{c}\text { Number of } \\
\text { torsion } \\
\text { modes }\end{array}$ & $\begin{array}{c}\text { Stall model, } \\
\text { MR/TR }\end{array}$ & $\begin{array}{c}\text { Wake } \\
\text { geometry }\end{array}$ \\
\hline & 0.525 & 6 & 2 & static/static & free \\
1 & 0.525 & 6 & 2 & static/static & prescribed \\
2 & 0.525 & 6 & 2 & dynamic/none & prescribed \\
$3^{\mathrm{a}}$ & 0.525 & 6 & 2 & static/static & prescribed \\
4 & 0.525 & 6 & 2 & static/static & prescribed \\
5 & 0.525 & 6 & 2 & $\begin{array}{l}\text { static/static } \\
\text { dynamic/none }\end{array}$ & prescribed \\
6 & 0.525 & 6 & 2 & dybed \\
7 & & 6 & & &
\end{tabular}

$\mathrm{a}_{\text {Trimmed to measured power level. }}$ 
ORIGIMA: P.ST is

OF POOR QUALITY

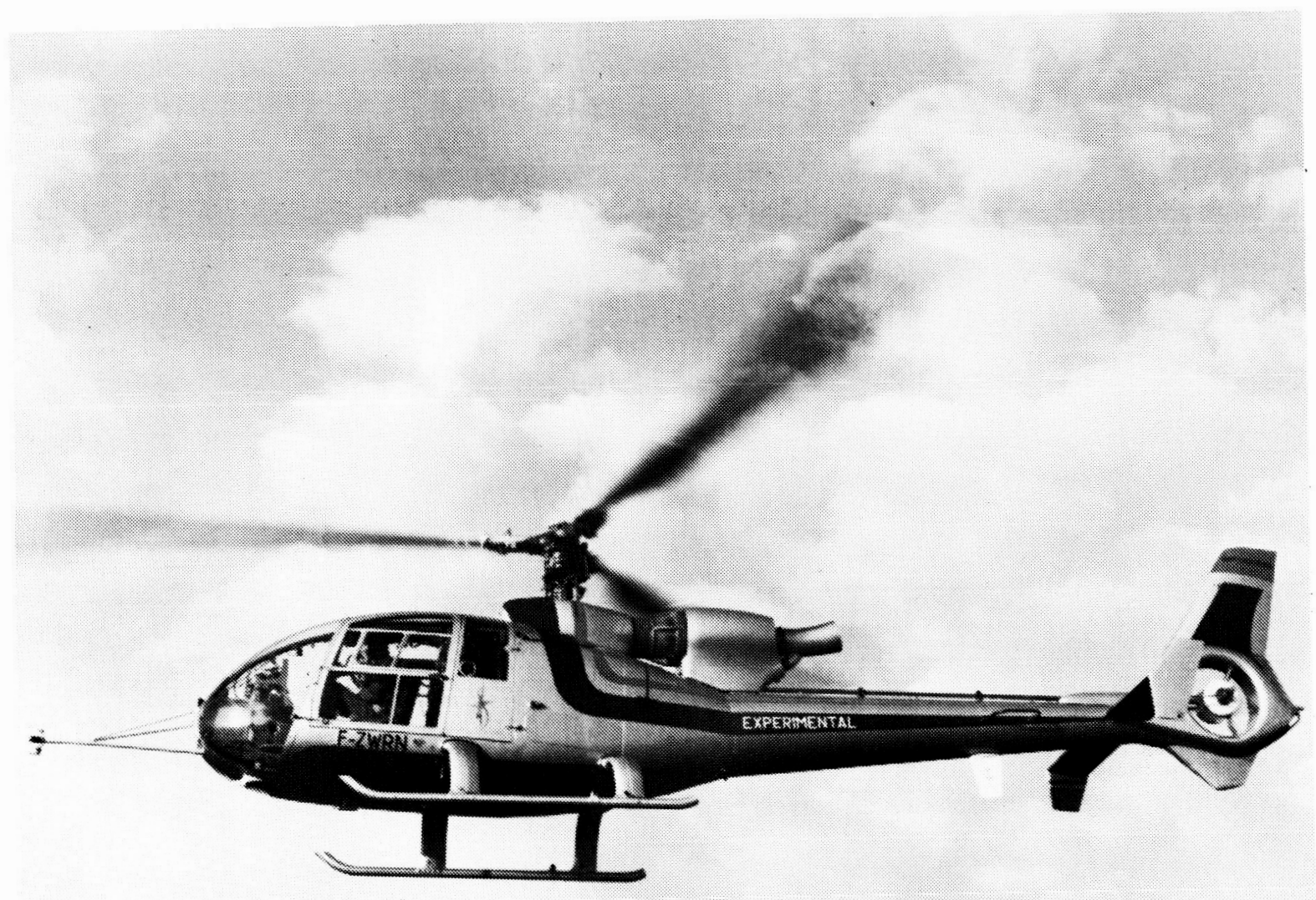

Fig. 1. SA349/2 Helicopter. 
ORIGITLL POS 19

OF POOR QUALTYY

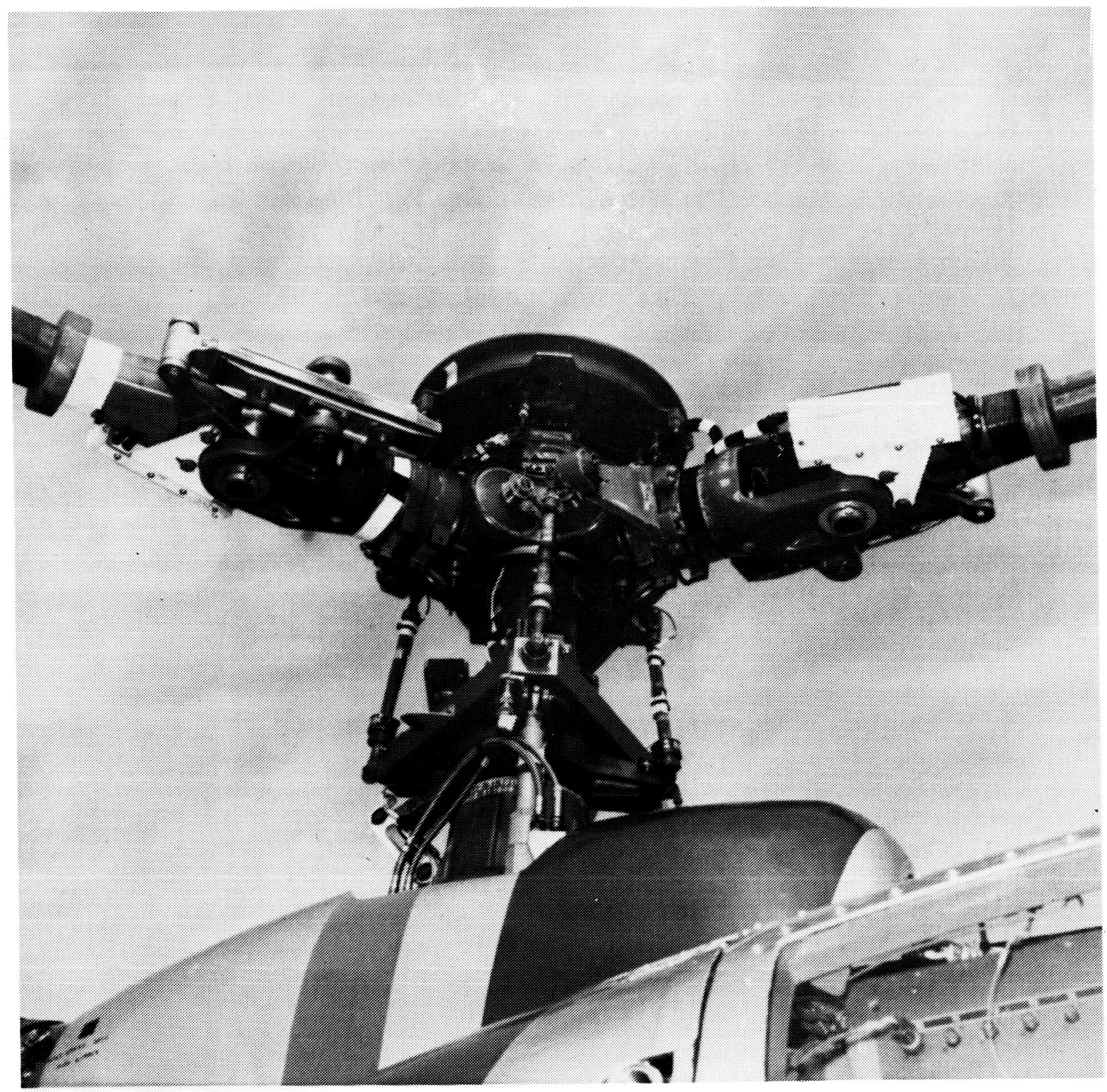

Fig. 2. Non Articule en Trainee (NAT) hub. 


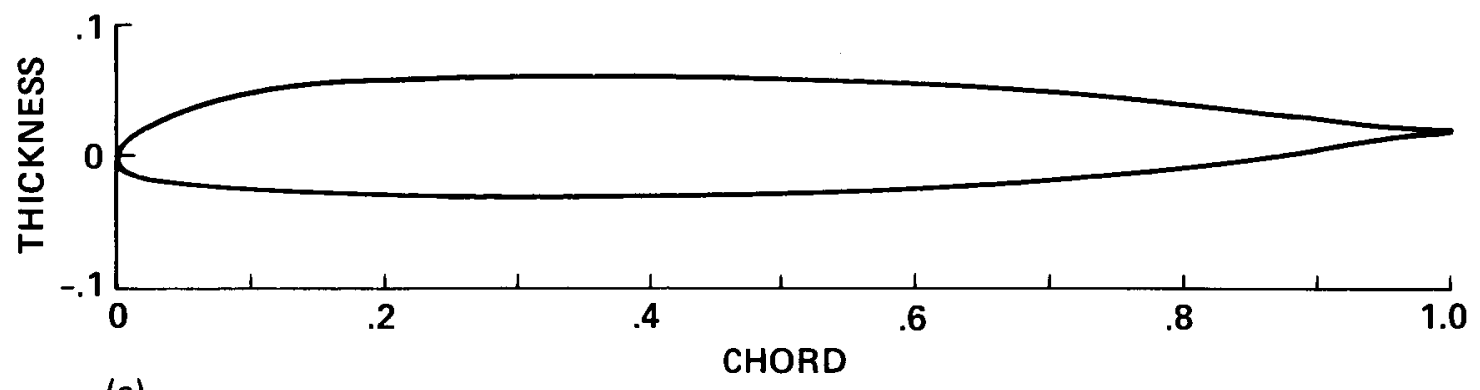

(a)
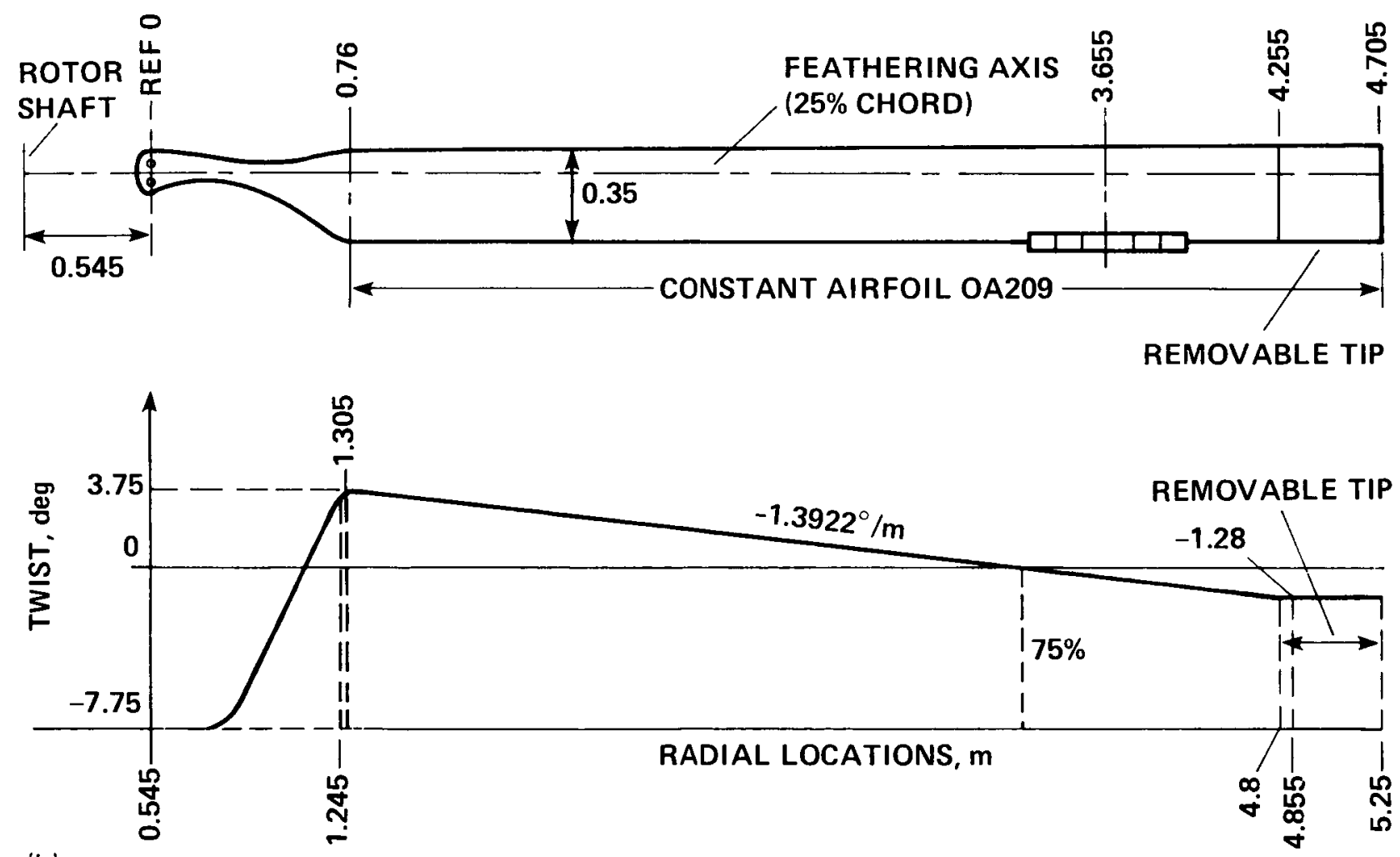

(b)

Fig. 3. Grande Vitesse (GV) blade characteristics. (a) 0A209 airfoil profile. (b) Blade twist distribution. 


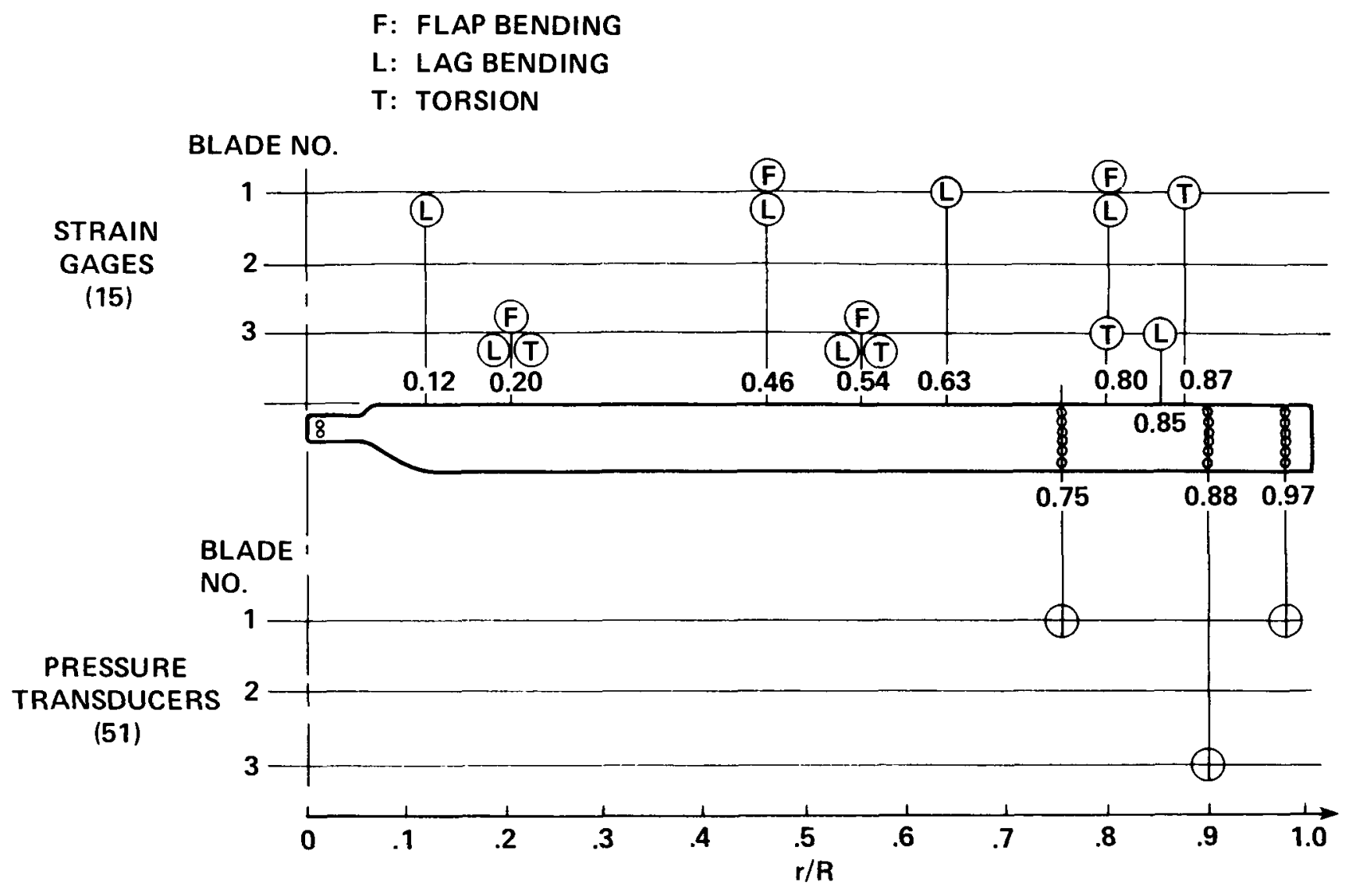

Fig. 4. Blade pressure transducer and strain gage locations. 


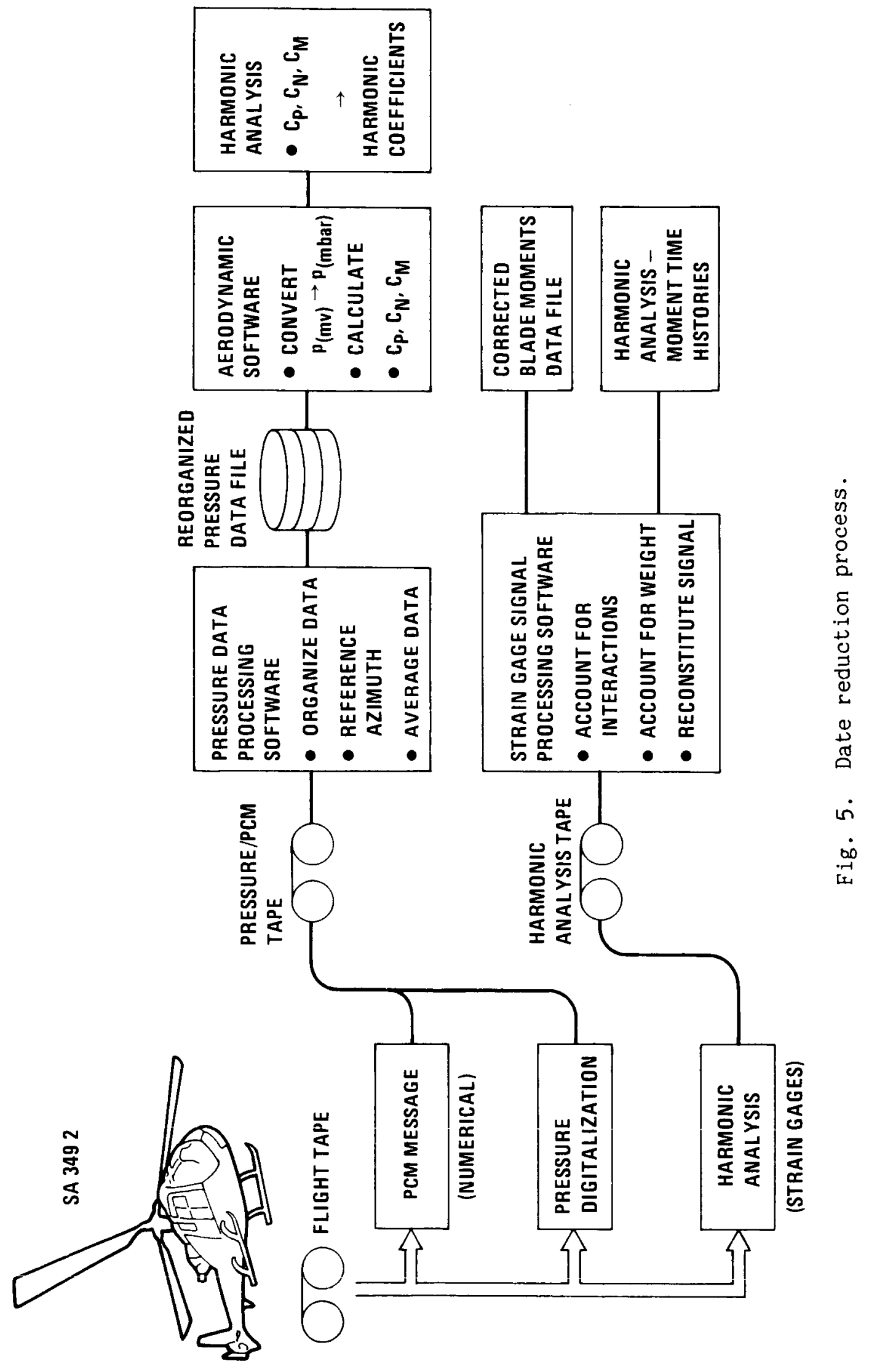




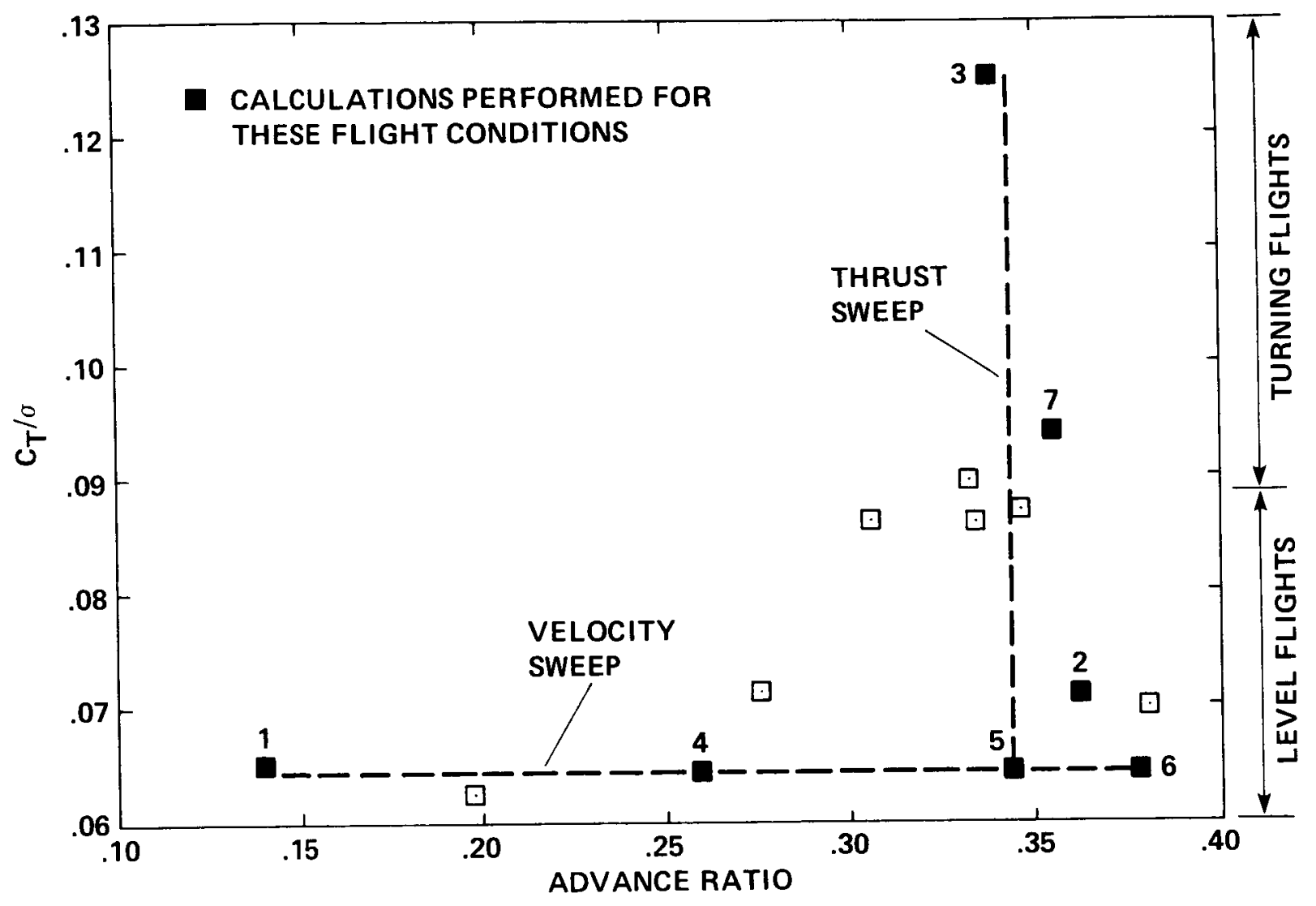

Fig. 6. Thrust and speed range of flight conditions. 
UNIFORM INFLOW

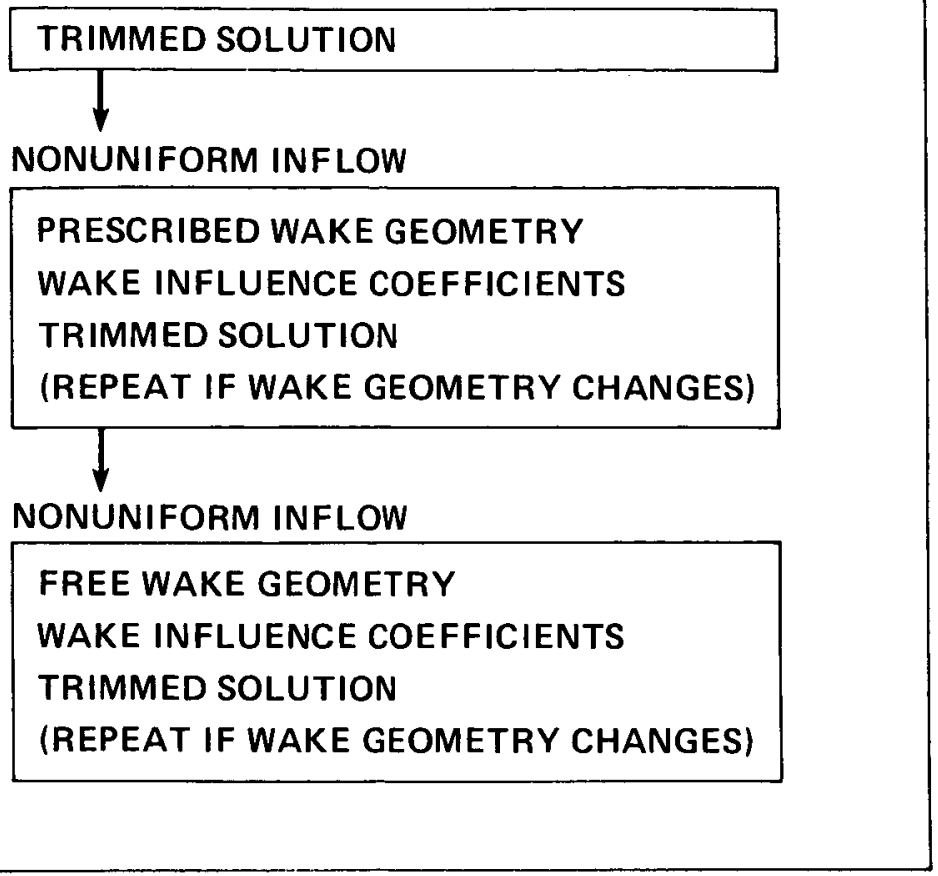

(a) Inflow Analysis Levels

\section{TRIMMED SOLUTION}

ITERATE CONTROLS TO TRIM
PERIODIC MOTION AND AIRLOADS

\begin{tabular}{|} 
CIRCULATION ITERATION \\
(INDUCED VELOCITY FROM LOADING) \\
MOTION ITERATION \\
ROTOR MOTION \\
AZIMUTHAL STEP \\
RADIAL INTEGRATION \\
SECTION AERODYNAMICS \\
UPDATE MOTION HARMONICS \\
TOTAL ROTOR LOADS \\
BODY VIBRATION \\
TEST MOTION CONVERGENCE \\
TEST CIRCULATION CONVERGENCE
\end{tabular}

TEST TRIM CONVERGENCE

(b) Trim, Circulation, and Motion Iterations

Fig. 7. CAMRAD solution procedure. 

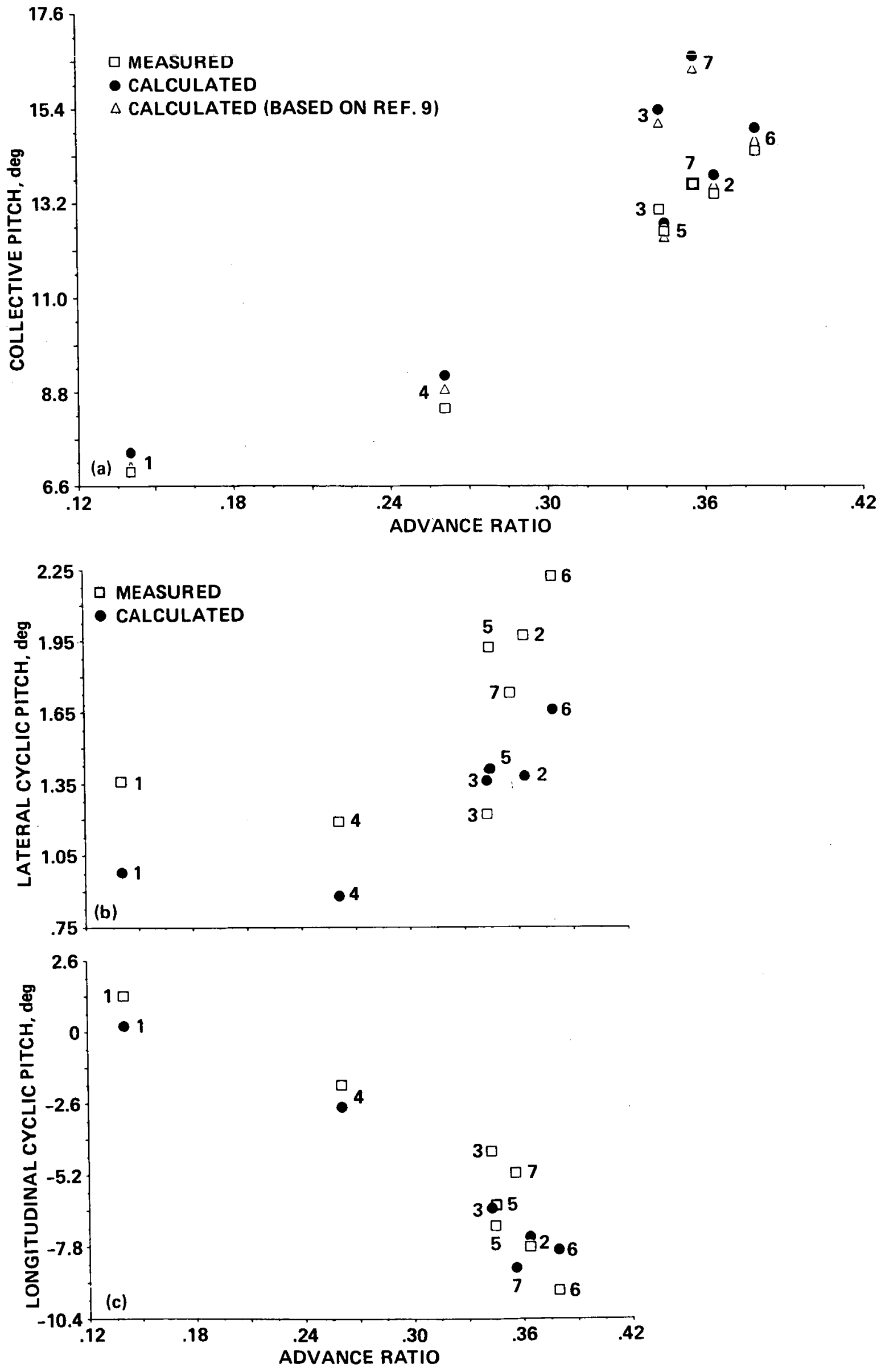

Fig. 8. Trimmed control inputs (numbers correspond to flight condition). (a) Collective. (b) Lateral cyclic. (c) Longitudinal cyclic. 


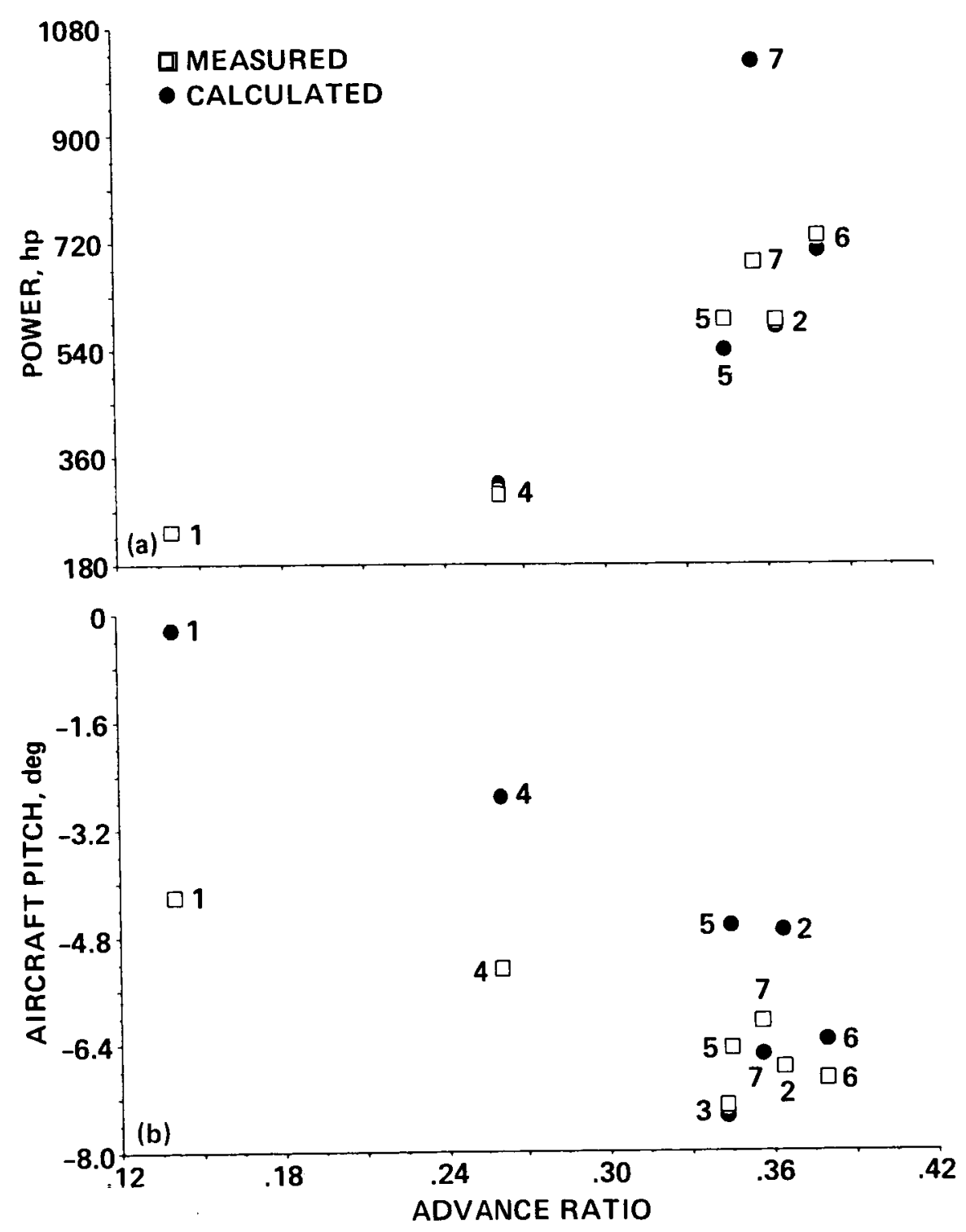

Fig. 9. Trimmed aircraft characteristics (numbers correspond to flight condition). (a) Performance. (b) Aircraft attitude. 

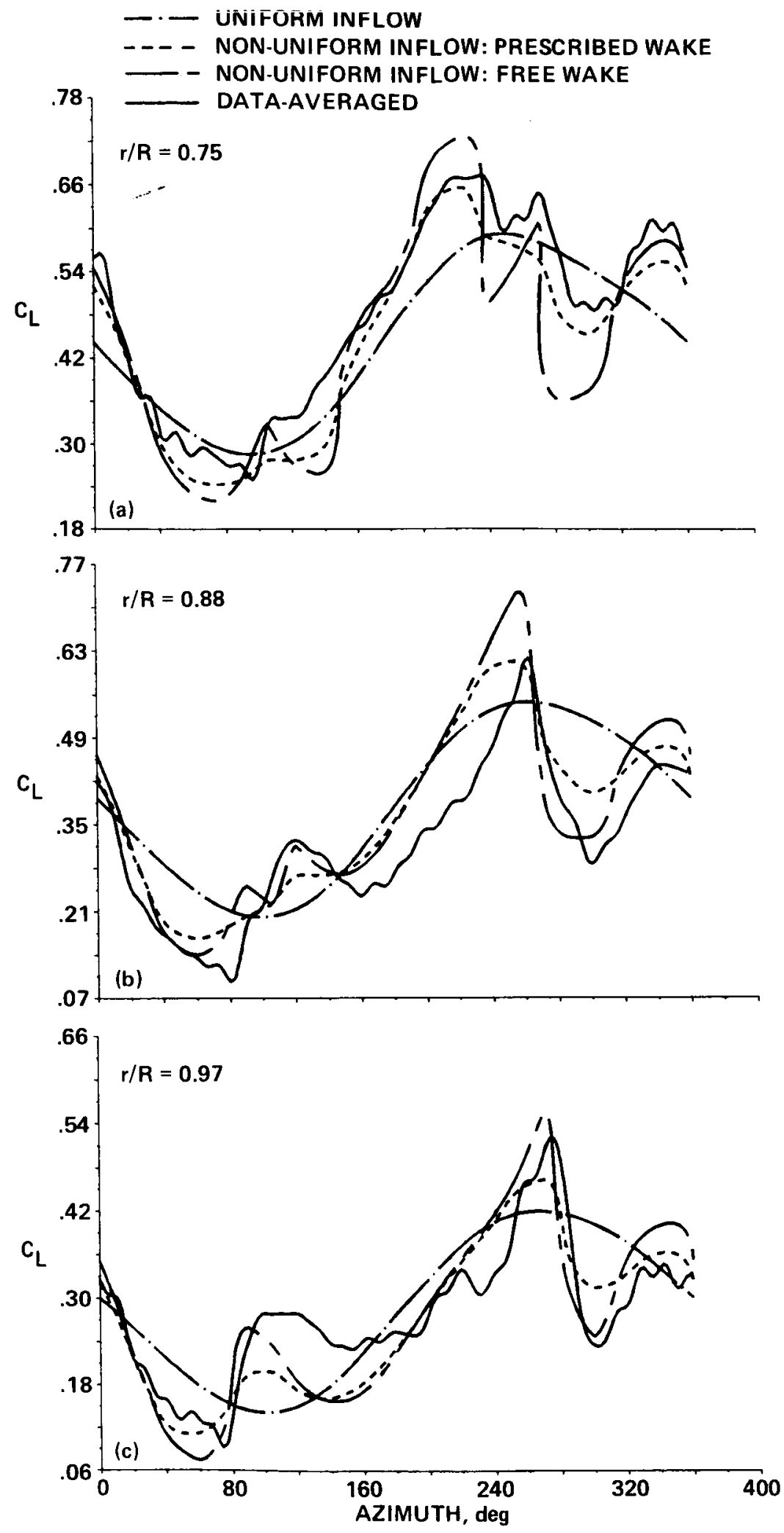

Fig. 10. Effect of wake geometry on lift - Condition 1. (a) $r / R=0.75$. (b) $r / R=0.88$. (c) $r / R=0.97$. 


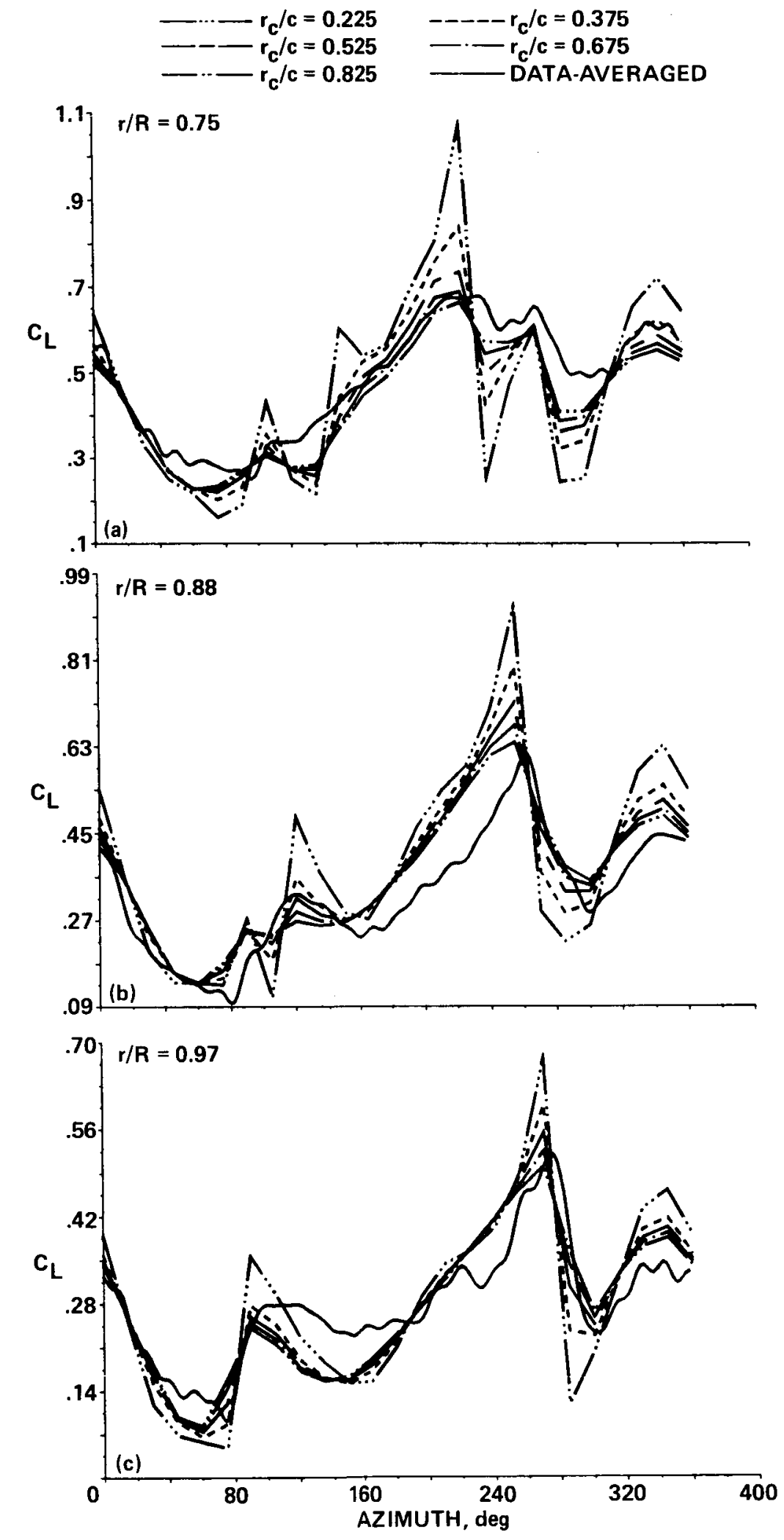

Eig. 11. Effect on lift of varying tip vortex core size - Condition 1. (a) $r / R=0.75$. (b) $r / R=0.88$. (c) $r / R=0.97$. 

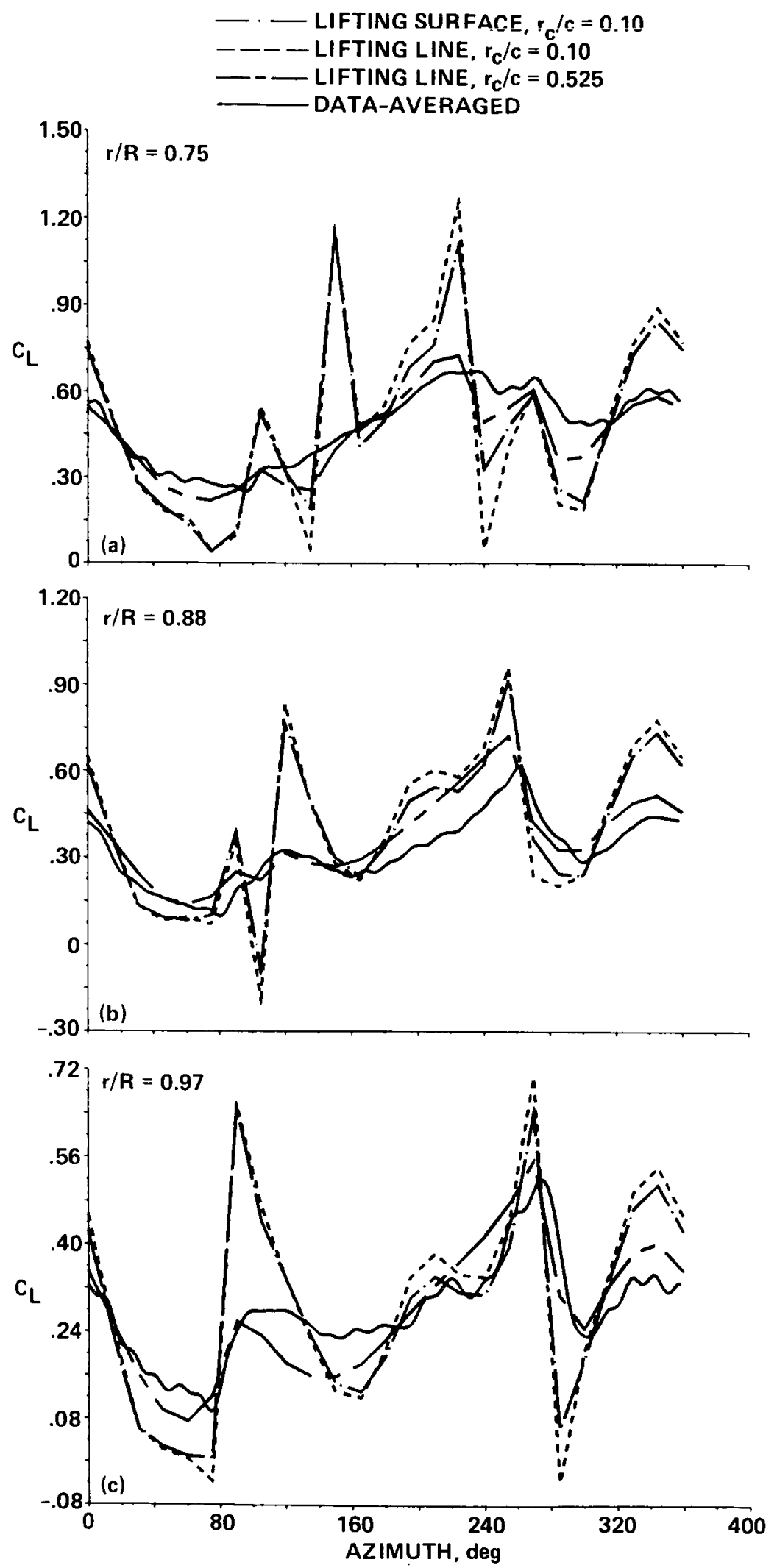

Fig. 12. Effect of lifting surface correction on lift - Condition 1. (a) $r / R \square 0.75$. (b) $r / R=0.88$. (c) $r / R=0.97$. 

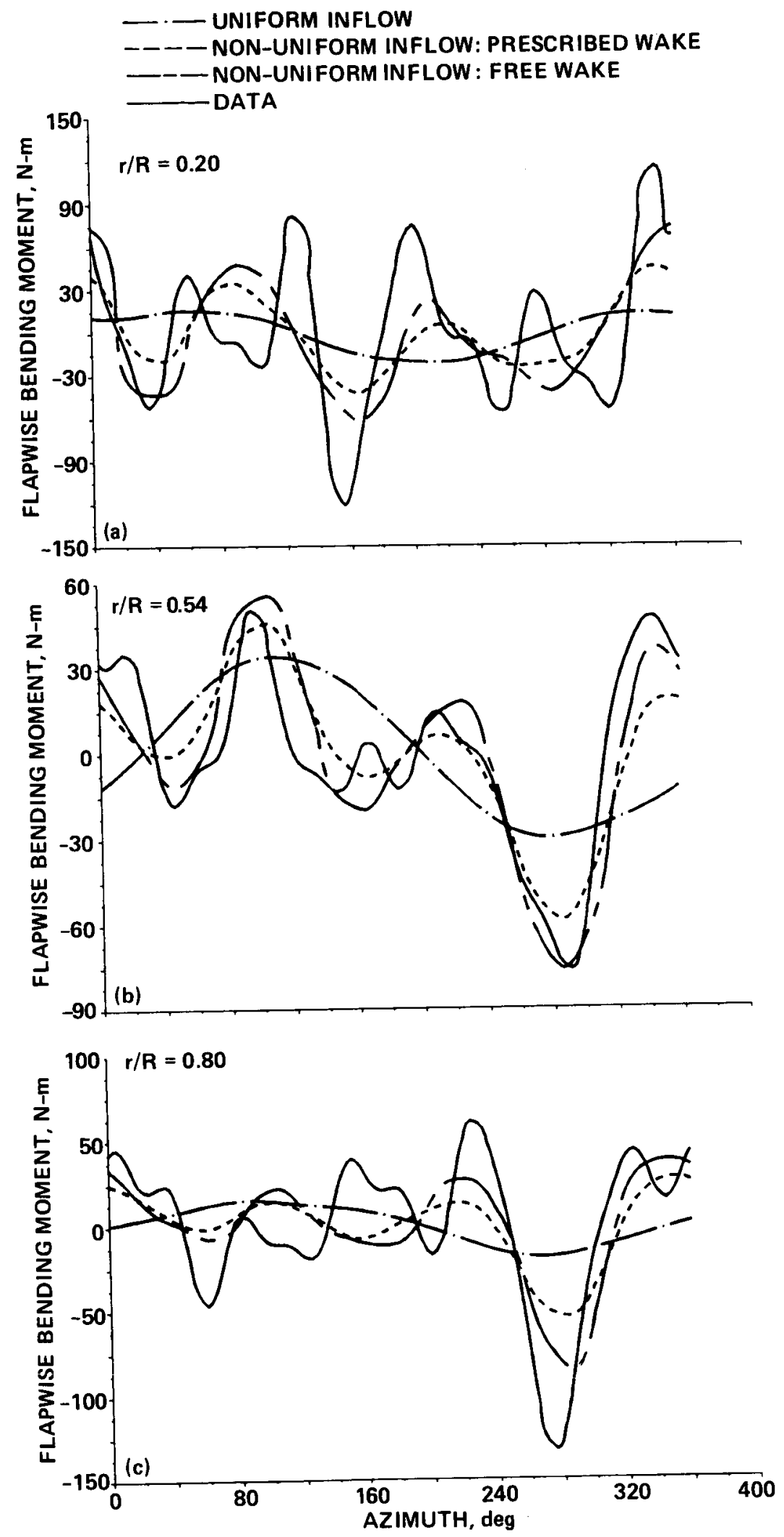

Fig. 13. Effect of wake geometry on flapwise bending moment - Condition 1. (a) $r / R=0.20$. (b) $r / R=0.54$. (c) $r / R=0.80$. 

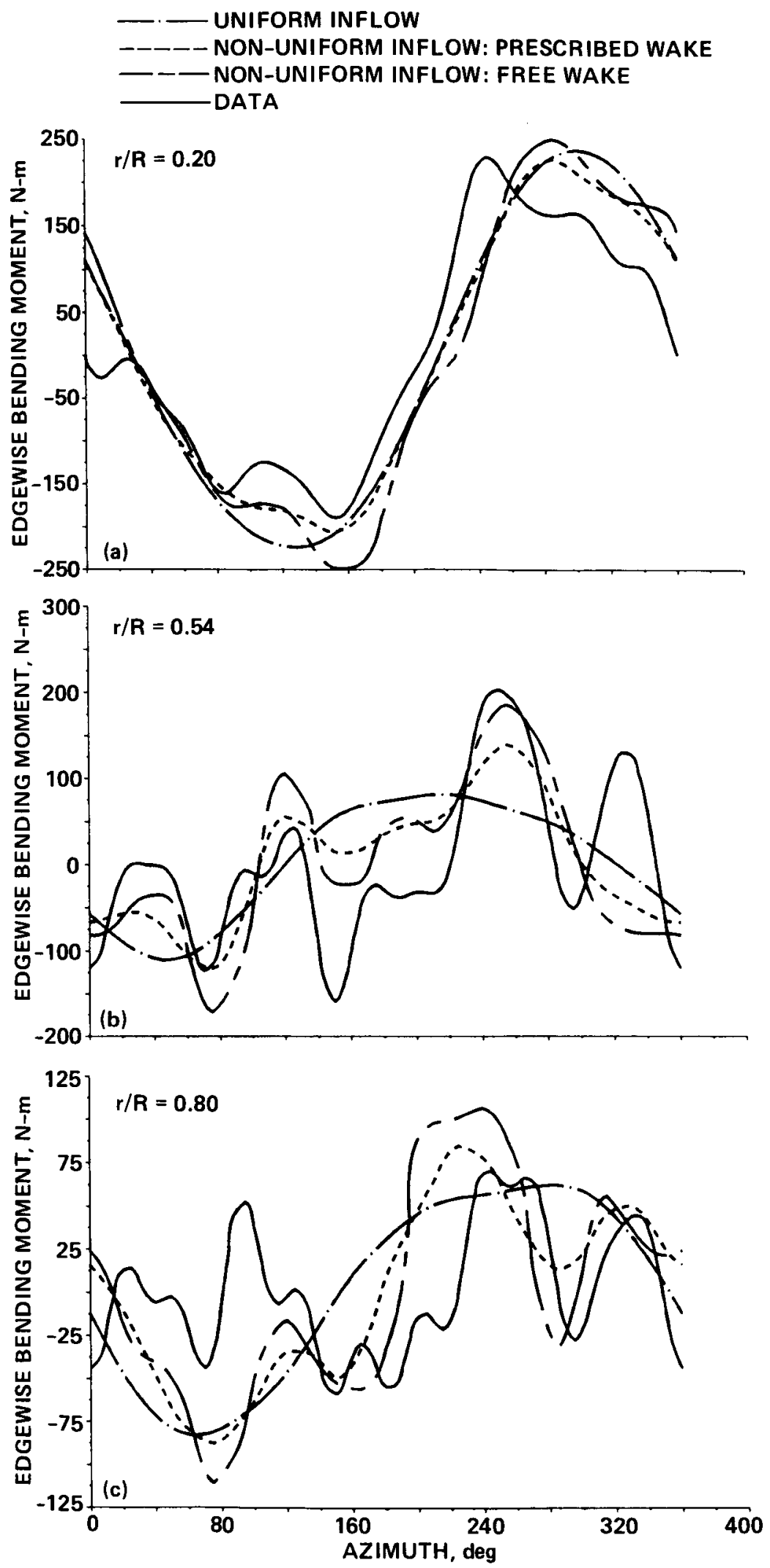

Fig. 14. Effect of wake geometry on edgewise bending moment - Condition 1. (a) $r / R=0.20$. (b) $r / R=0.54$. (c) $r / R=0.80$. 

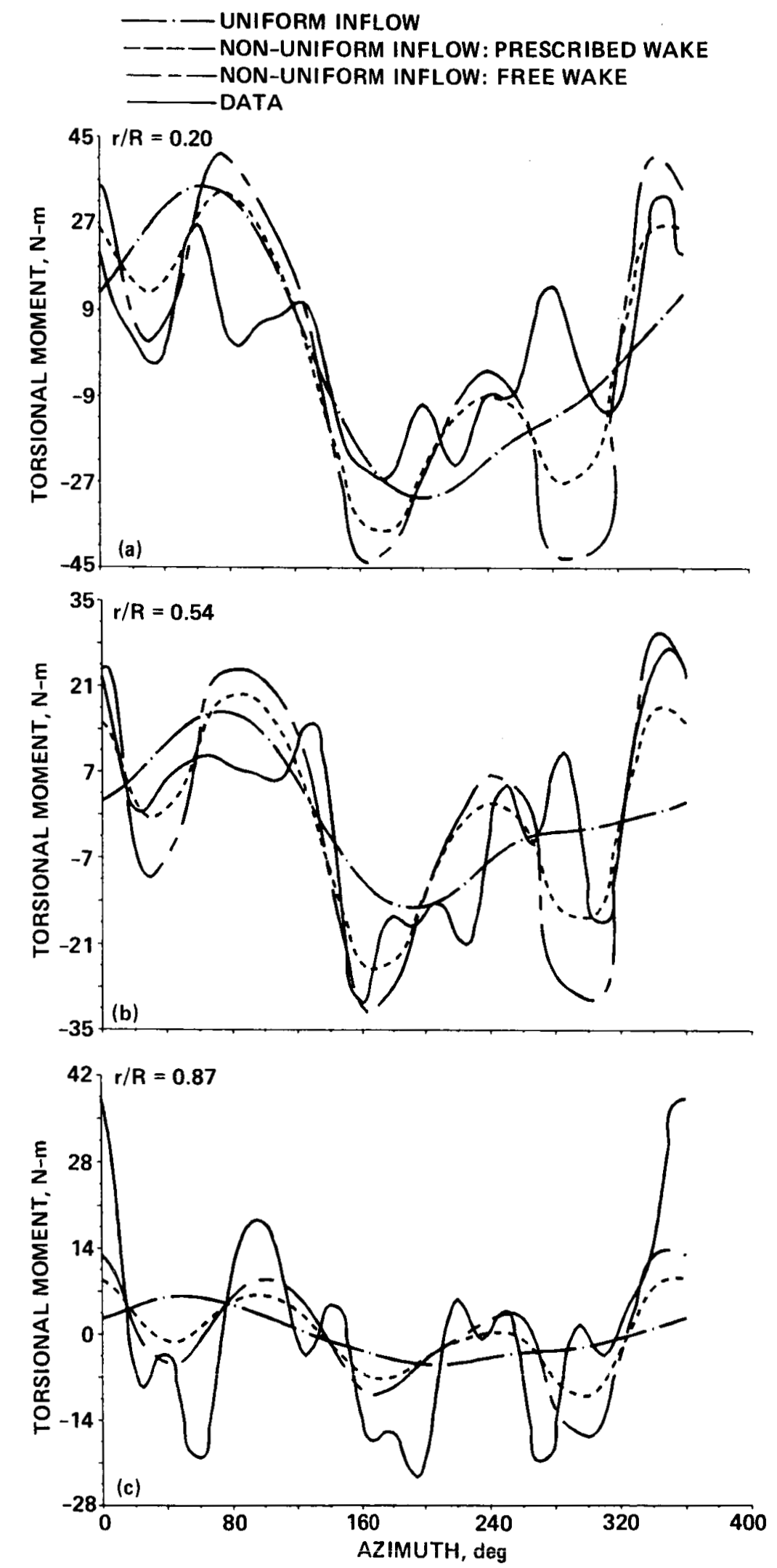

Fig. 15. Effect of wake geometry on torsional moment - Condition 1. (a) $r / R=0.20$. (b) $r / R=0.54$. (c) $r / R=0.87$. 


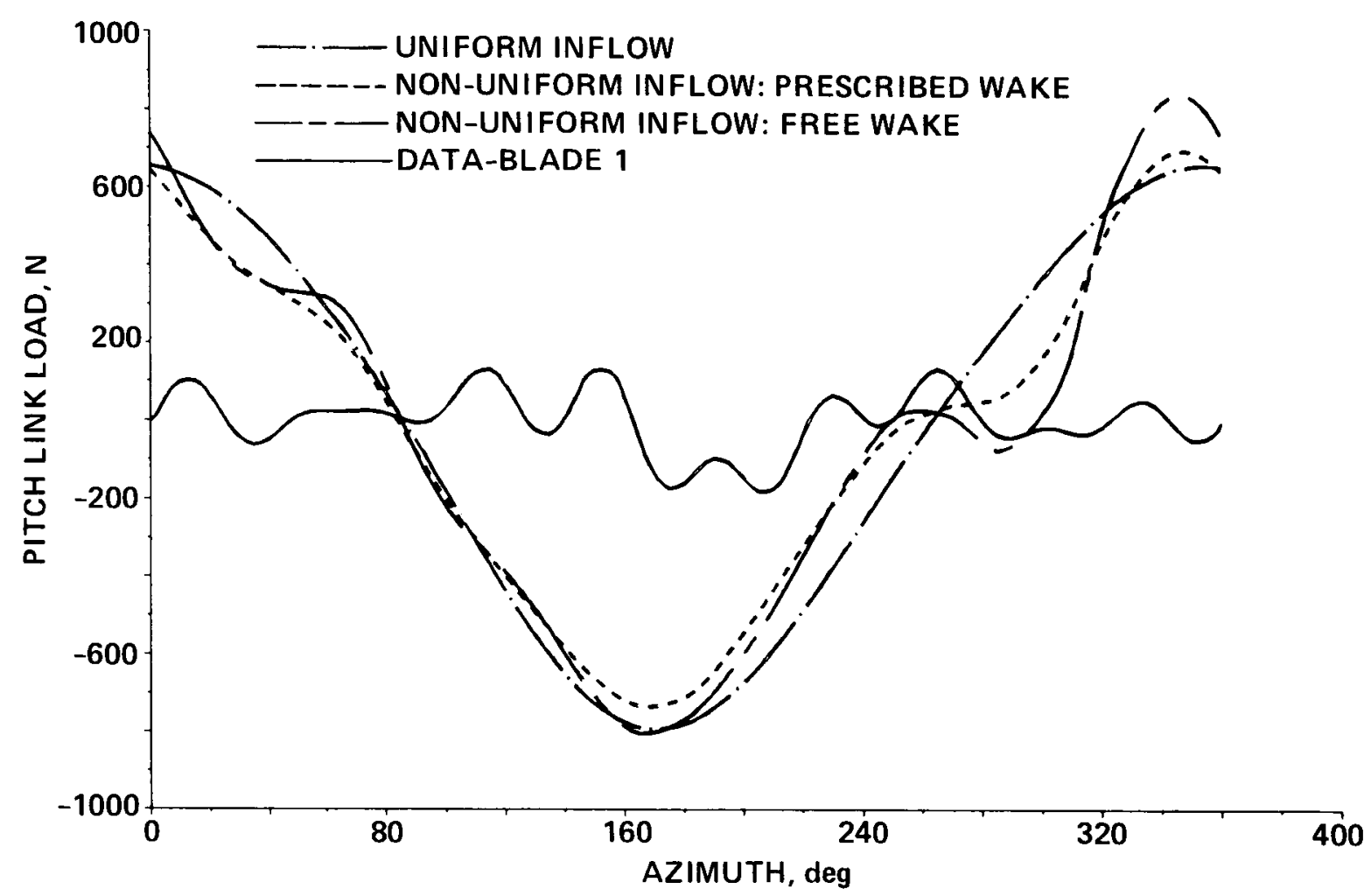

Fig. 16. Effect of wake geometry on pitch-link loads - Condition 1. 

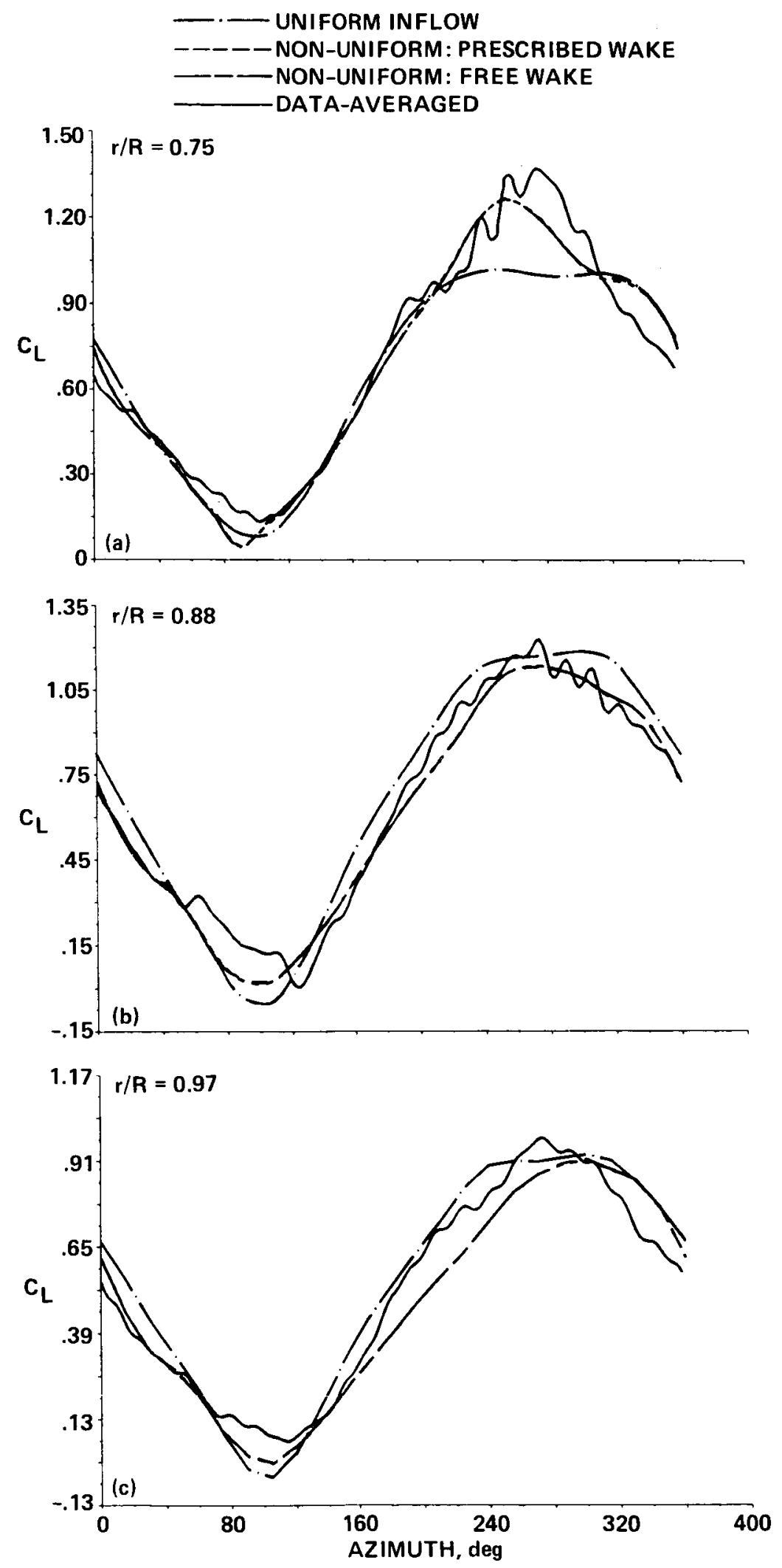

Fig. 17. Effect of wake geometry on lift - Condition 2 . (a) $r / R=0.75$. (b) $r / R=0.88$. (c) $r / R=0.97$. 

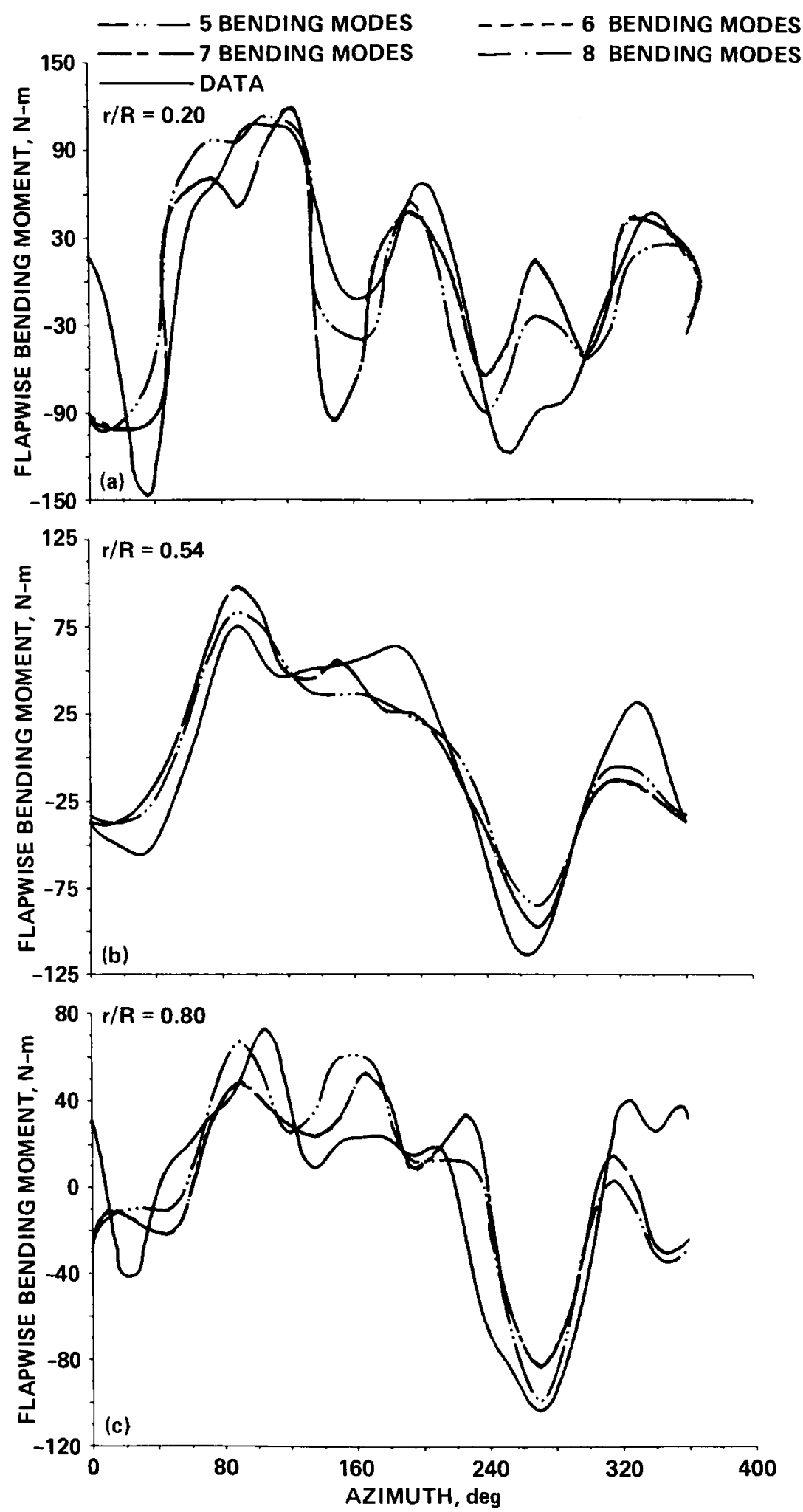

Fig. 18. Effect of number of bending modes on flapwise bending moment Condition 2. (a) $r / R=0.20$. (b) $r / R=0.54$. (c) $r / R=0.80$. 

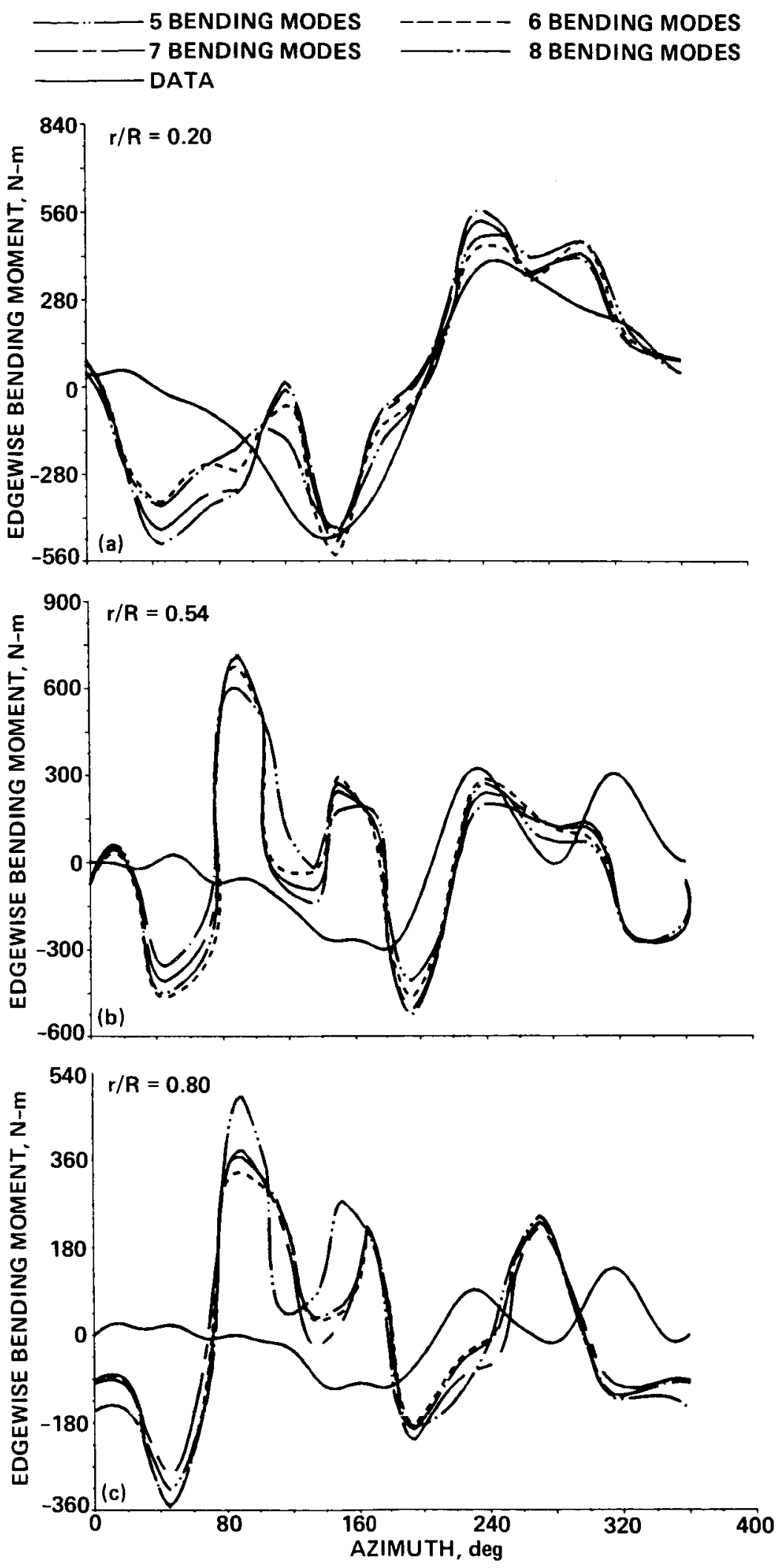

Fig. 19. Effect of number of bending modes on edgewise bending moment Condition 2. (a) $r / R=0.20$. (b) $r / R=0.54$. (c) $r / R=0.80$. 


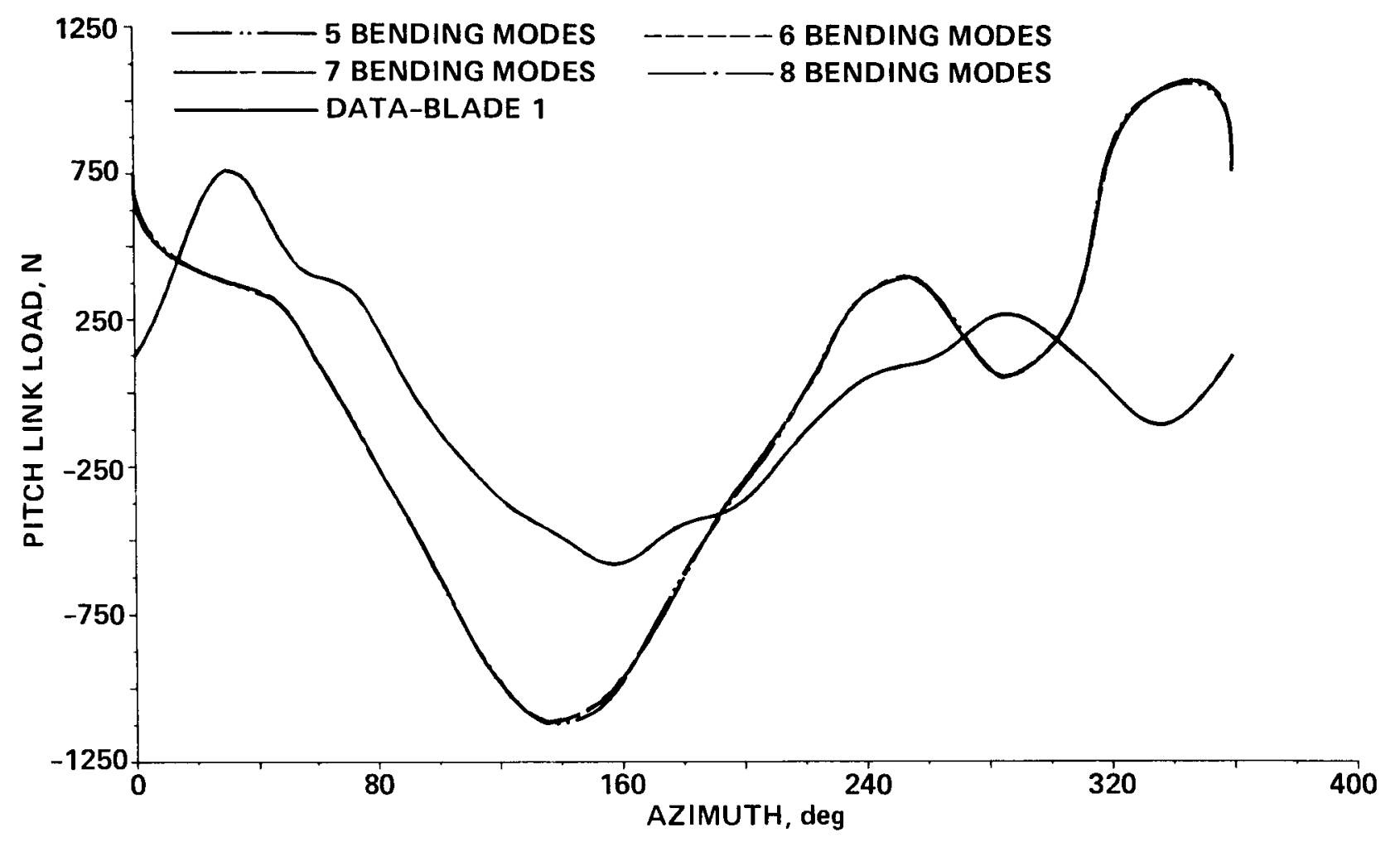

Eig. 20. Effect of number of bending modes on pitch-link loads Condition 2. 

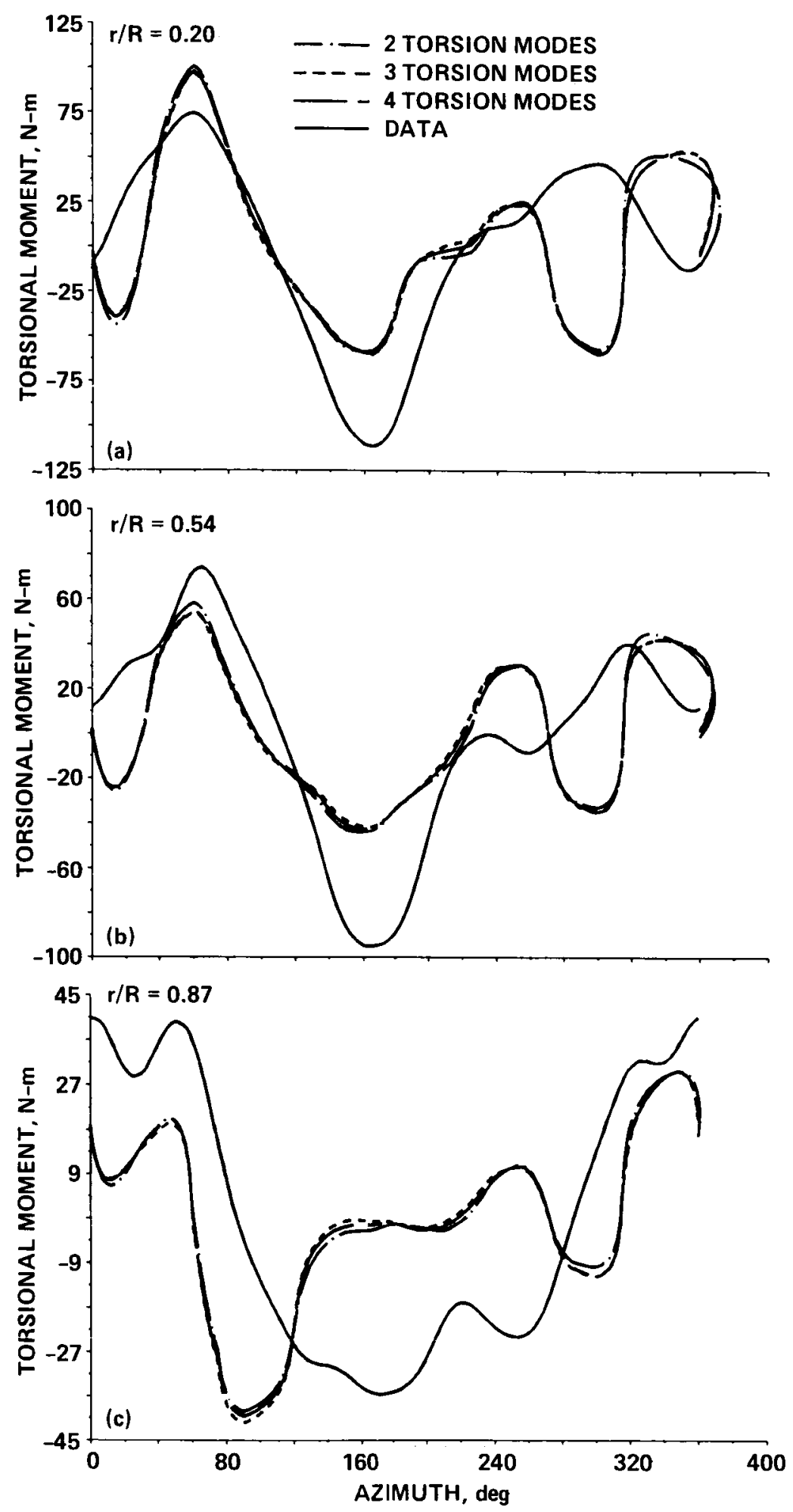

Fig. 21. Effect of number of torsion modes on torsional moment Condition 2. (a) $r / R \square 0.20$. (b) $r / R=0.54$. (c) $r / R=0.87$. 

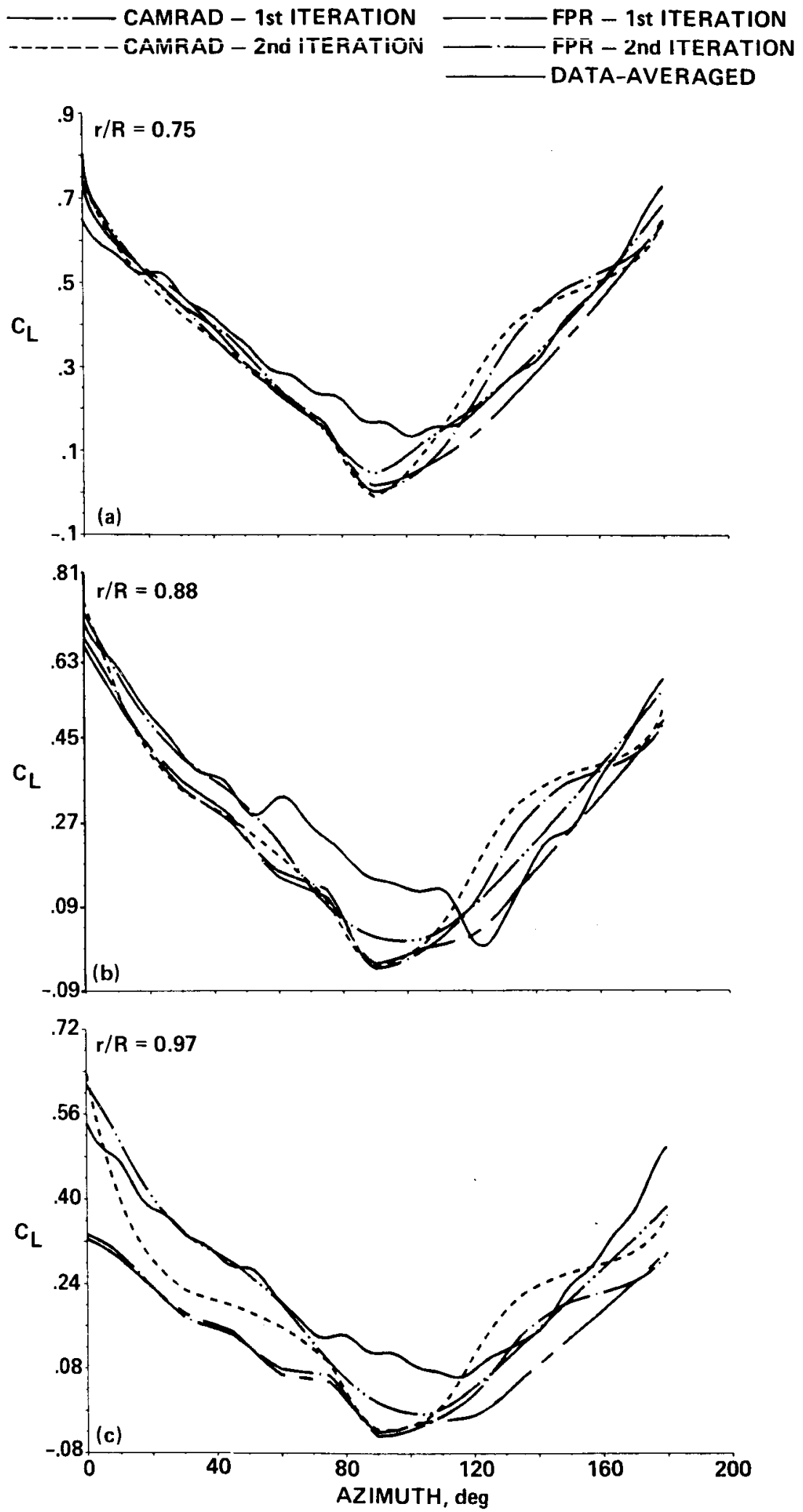

Fig. 22. Lift predictions using CAMRAD and CAMRAD/FPR - Condition 2. (a) $r / R=0.75$. (b) $r / R=0.88$. (c) $r / R=0.97$. 


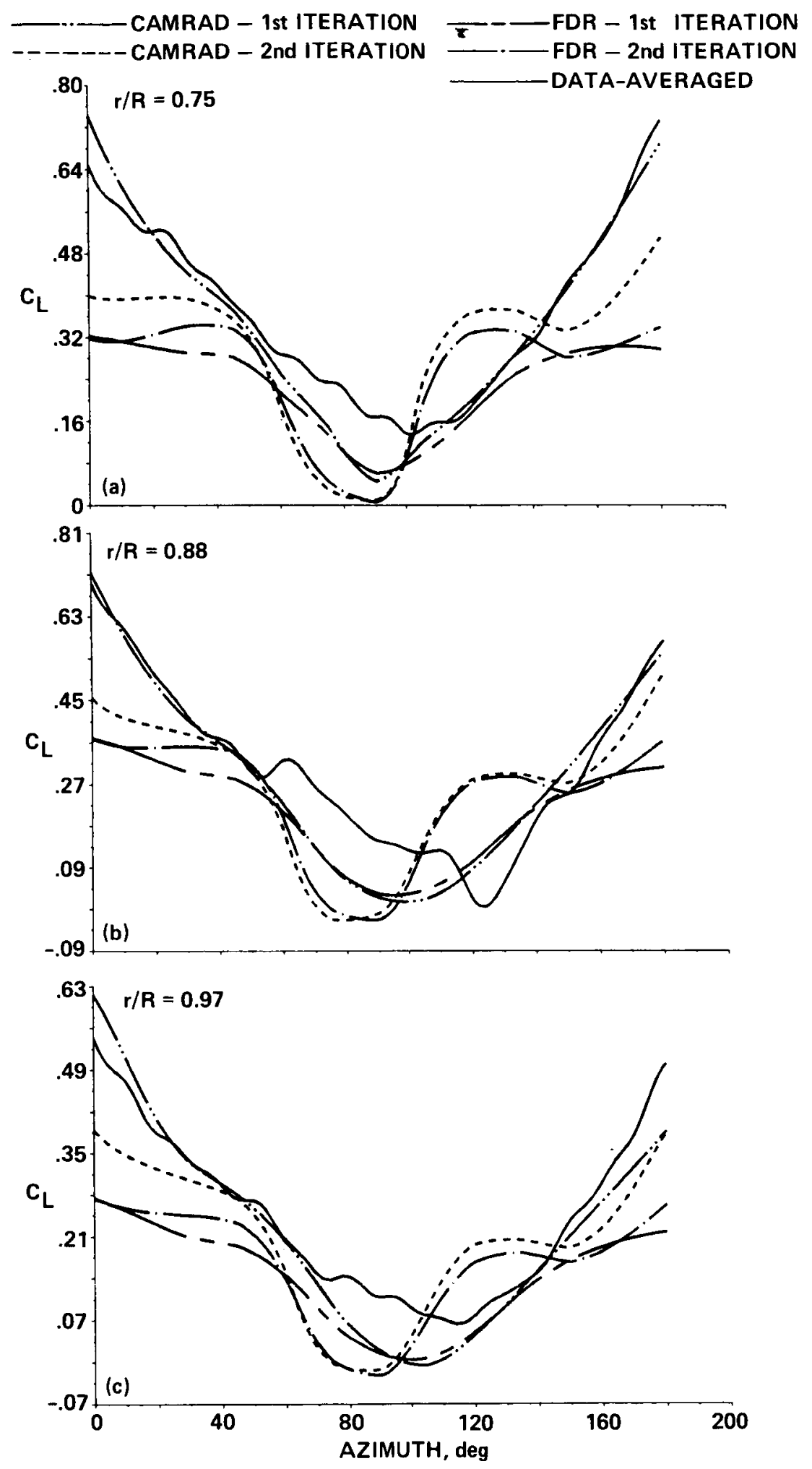

Fig. 23. Lift predictions using CAMRAD and CAMRAD/FDR - Condition 2. (a) $r / R=0.75$. (b) $r / R=0.88$. (c) $r / R=0.97$. 

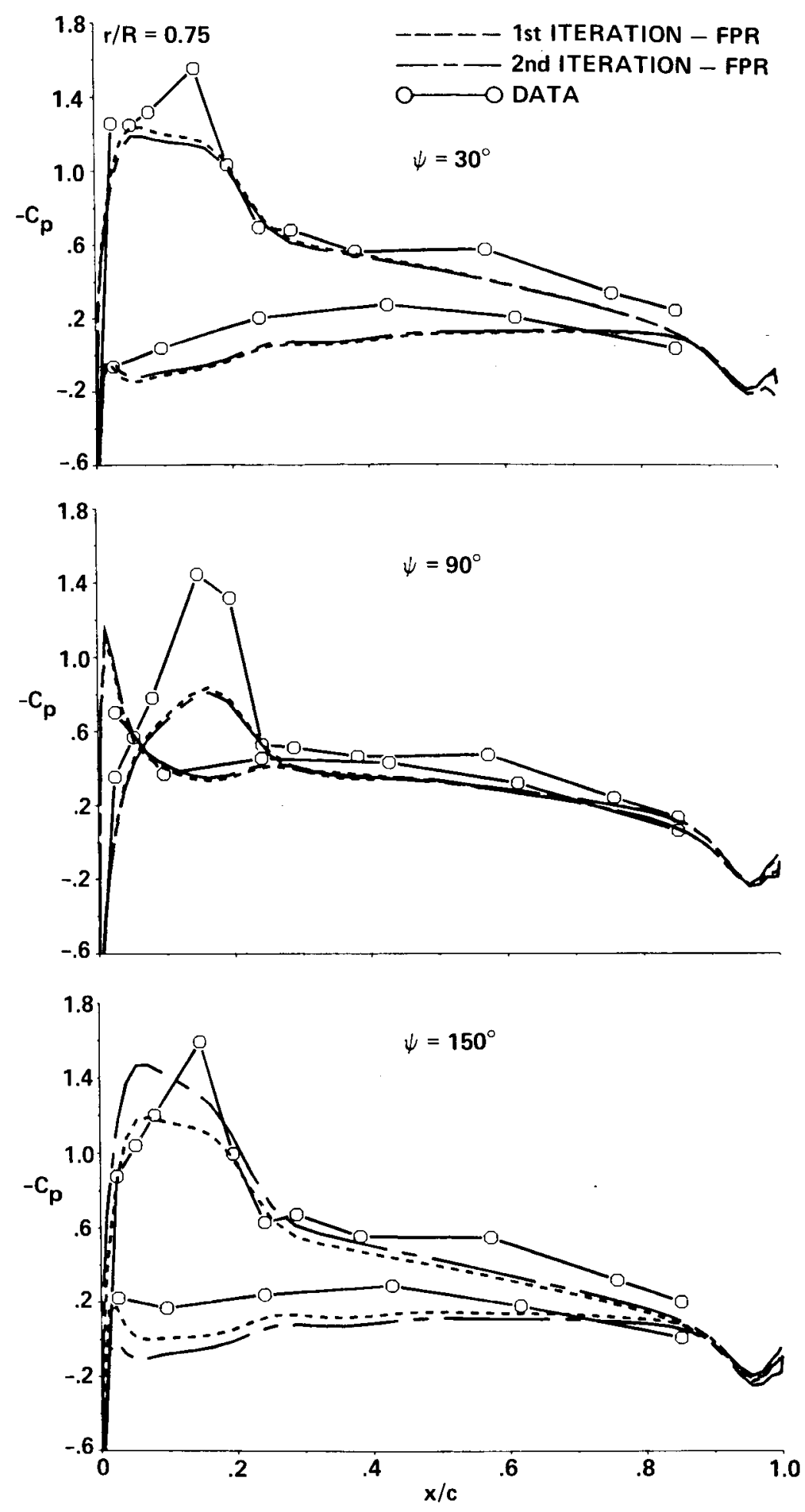

(a) $r / R=0.75:$ azimuth $=30,90,150$ degrees.

Fig. 24. FPR surface pressure coefficient predictions - Condition 2. 

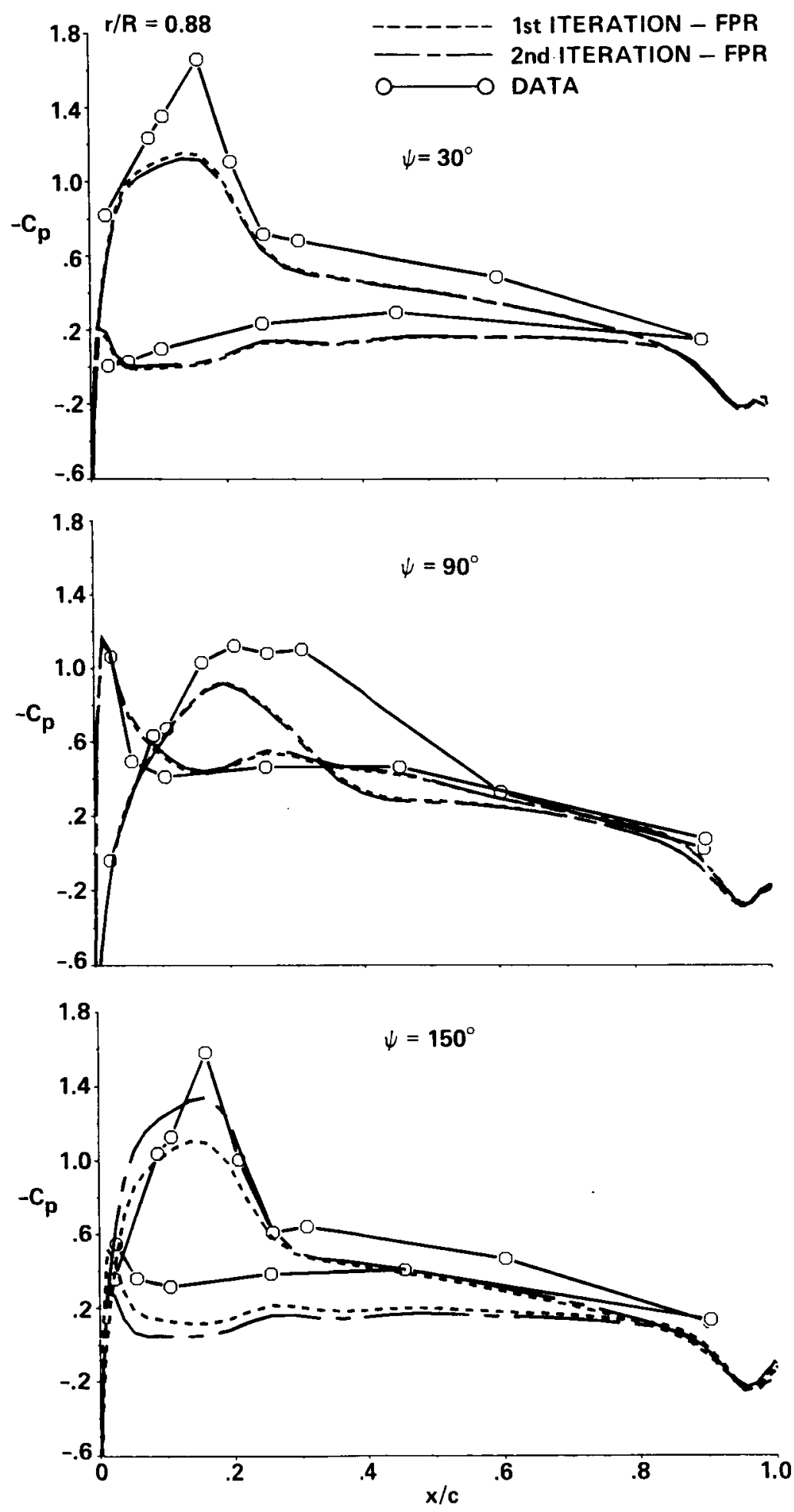

(b) $r / R$ 0.88: azimuth $=30,90,150$ degrees.

Fig. 24. Continued. 

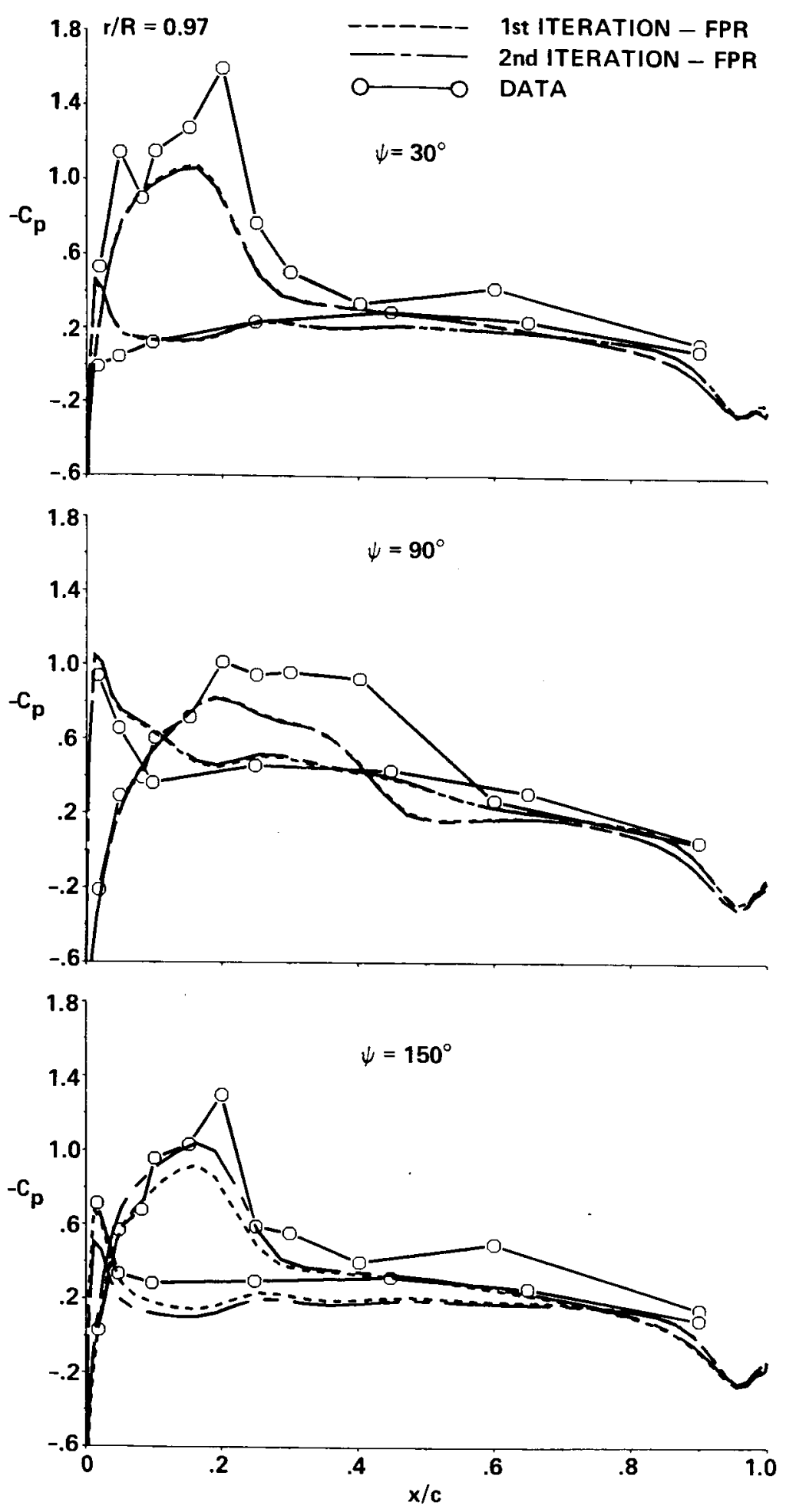

(c) $r / R$ a $0.97:$ azimuth $-30,90,150$ degrees.

Fig. 24. Concluded. 

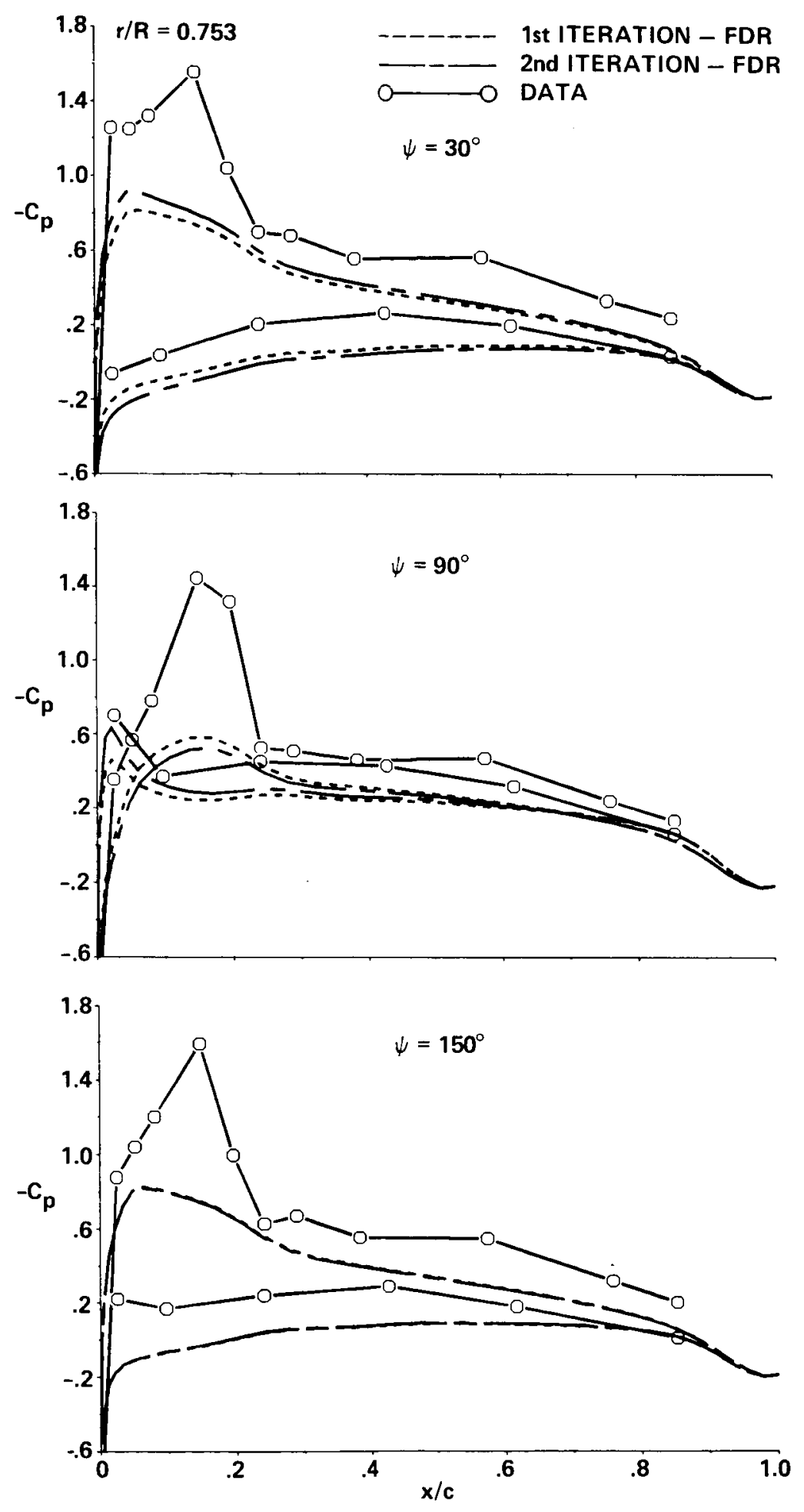

(a) $r / R$ a 0.753: azimuth $=30,90,150$ degrees.

Fig. 25. FDR surface pressure coefficient predictions - Condition 2. 

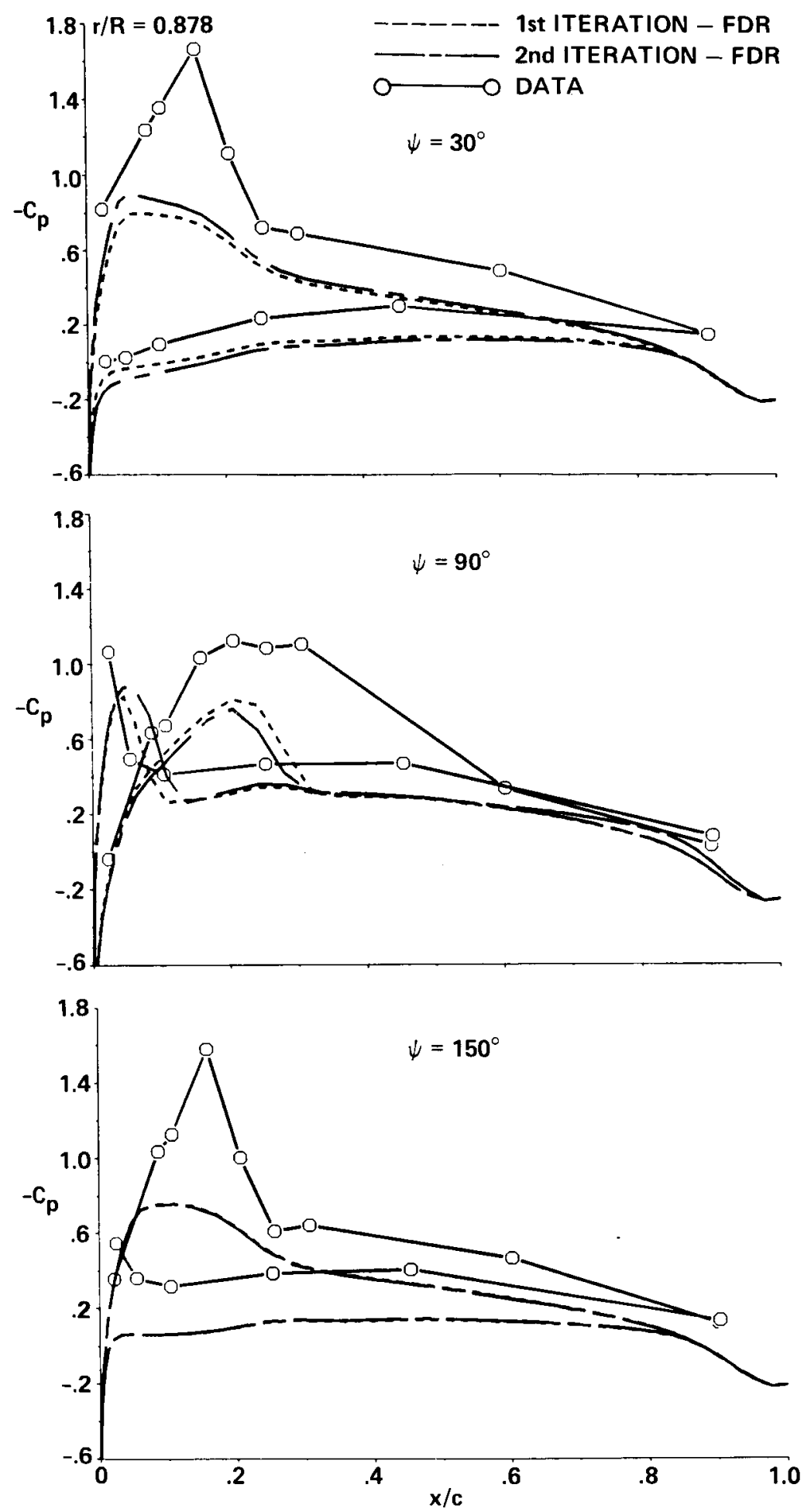

(b) $r / R=0.878:$ azimuth $=30,90,150$ degrees.

Fig. 25. Continued. 

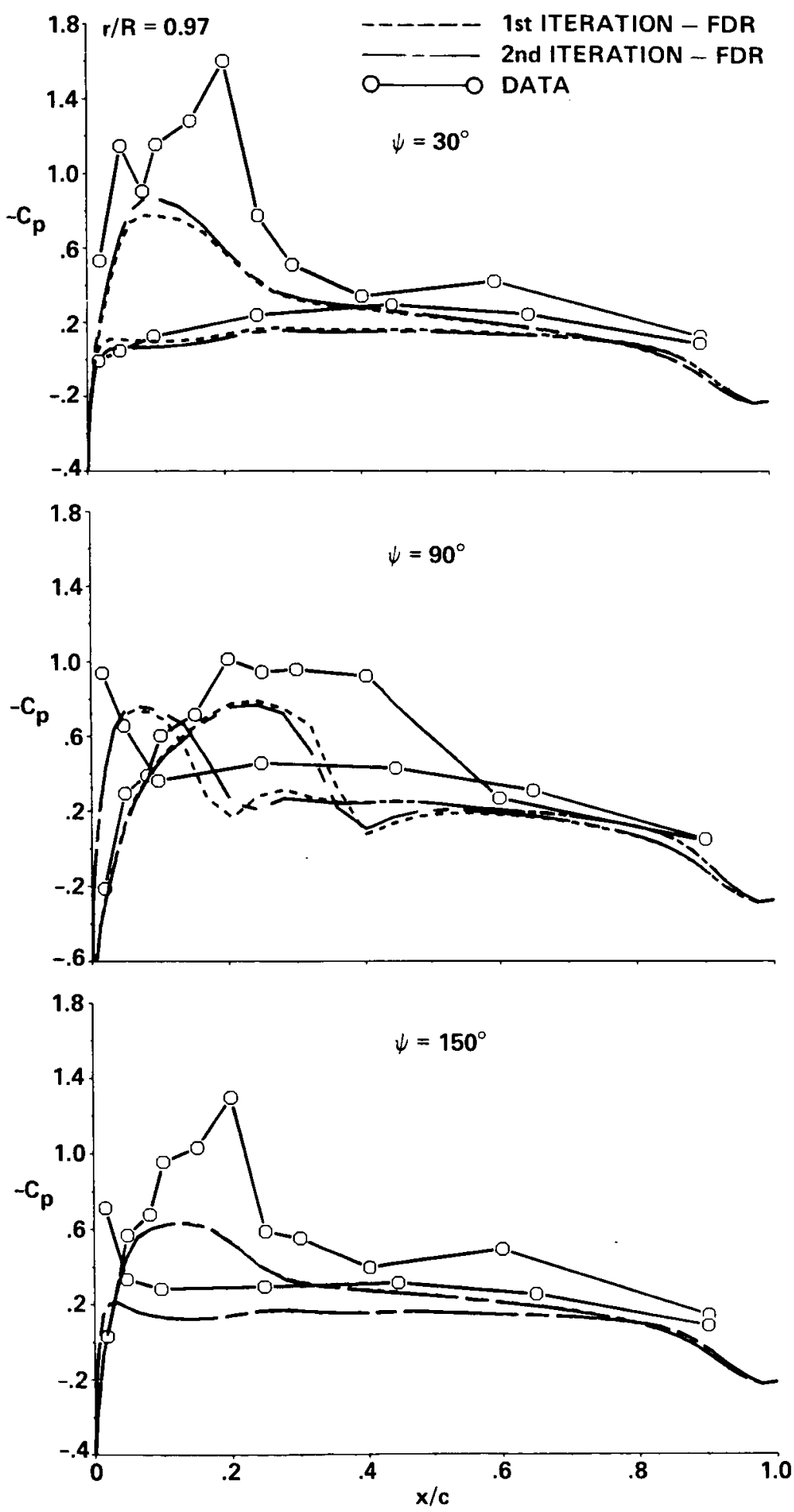

(c) $r / R=0.97:$ azimuth $=30,90,150$ degrees.

Fig. 25. Concluded. 

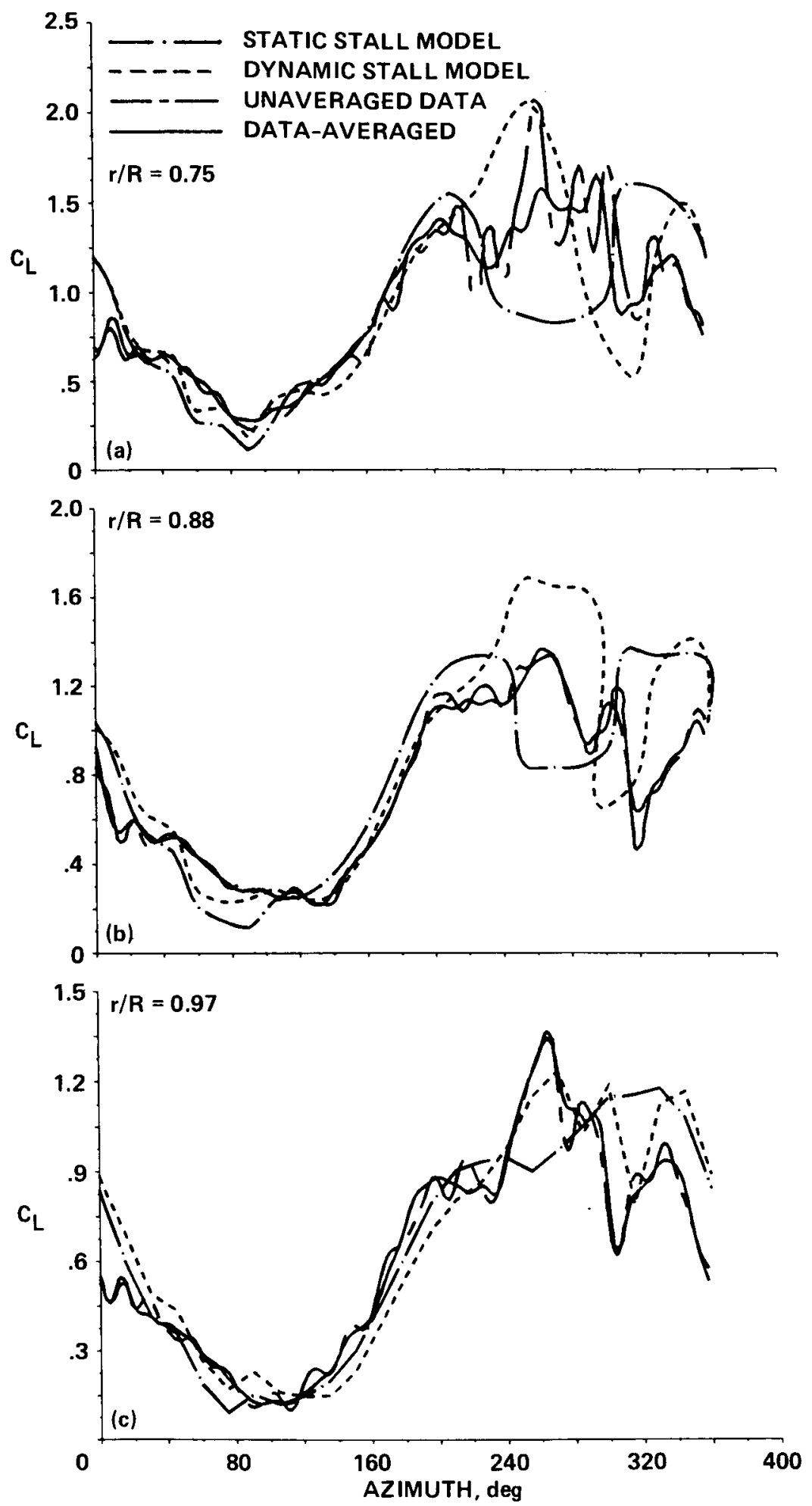

Fig. 26. Effect of stall model on lift - Condition 3. (a) $r / R=0.75$. (b) $r / R=0.88$. (c) $r / R=0.97$. 

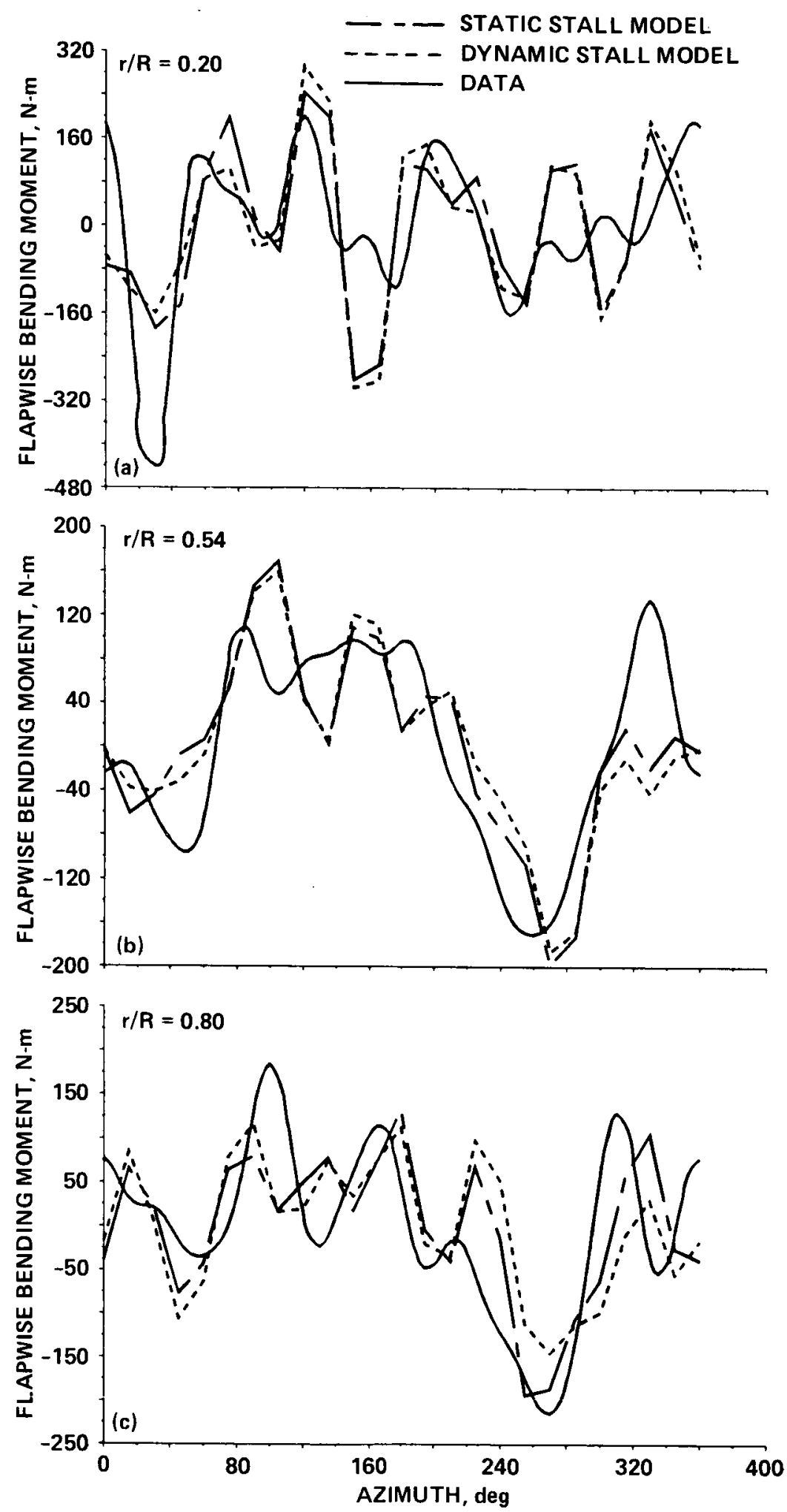

Fig. 27. Effect of stall model on flapwise bending moment - Condition 3. (a) $r / R=0.20$. (b) $r / R=0.54$. (c) $r / R=0.80$. 

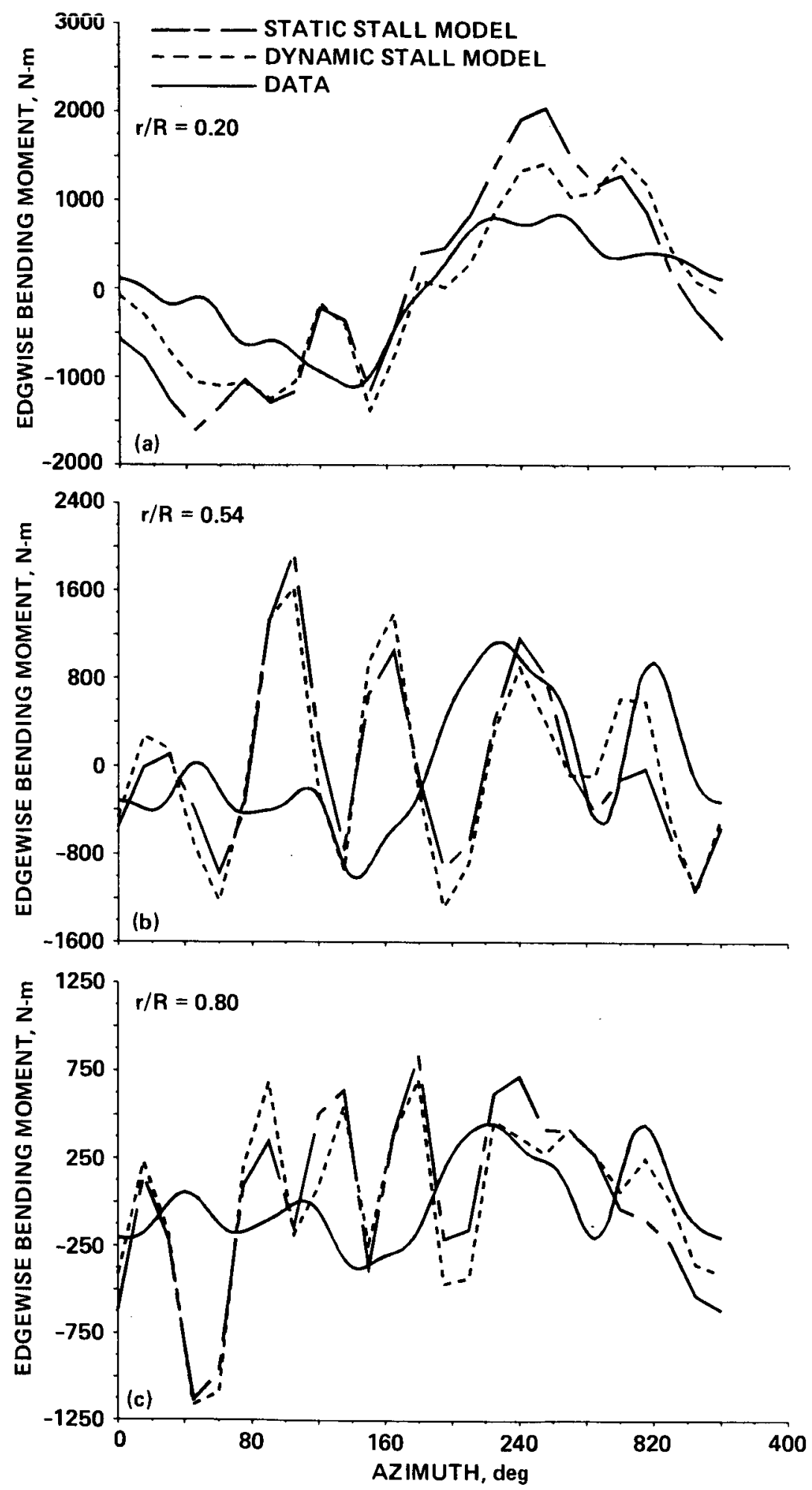

Fig. 28. Effect of stall model on edgewise bending moment - Condition 3. (a) $r / R=0.20$. (b) $r / R=0.54$. (c) $r / R=0.80$. 


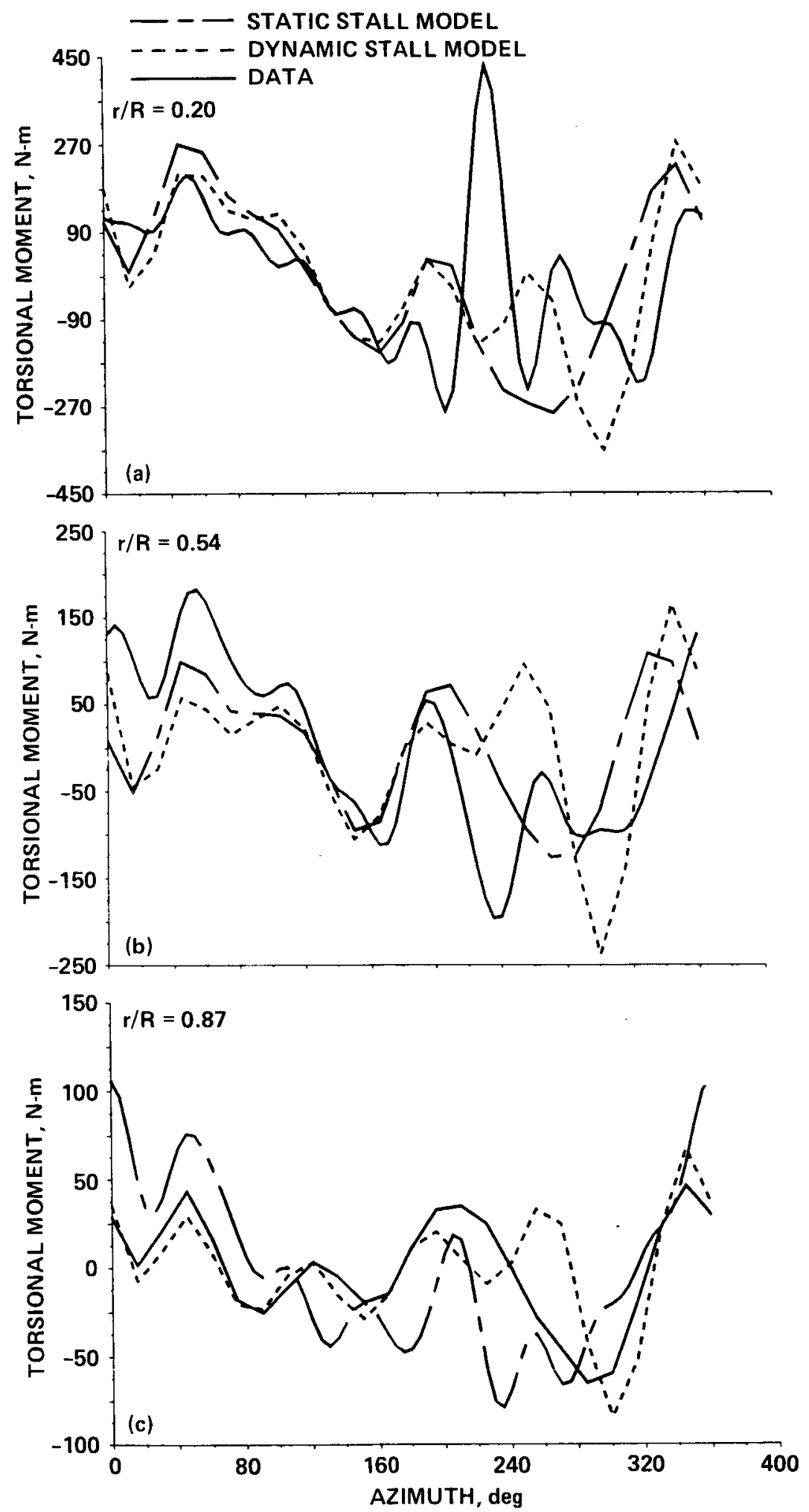

Fig. 29. Effect of stall model on torsional moment - Condition 3 . (a) $r / R=0.20$. (b) $r / R=0.54$. (c) $r / R=0.87$. 


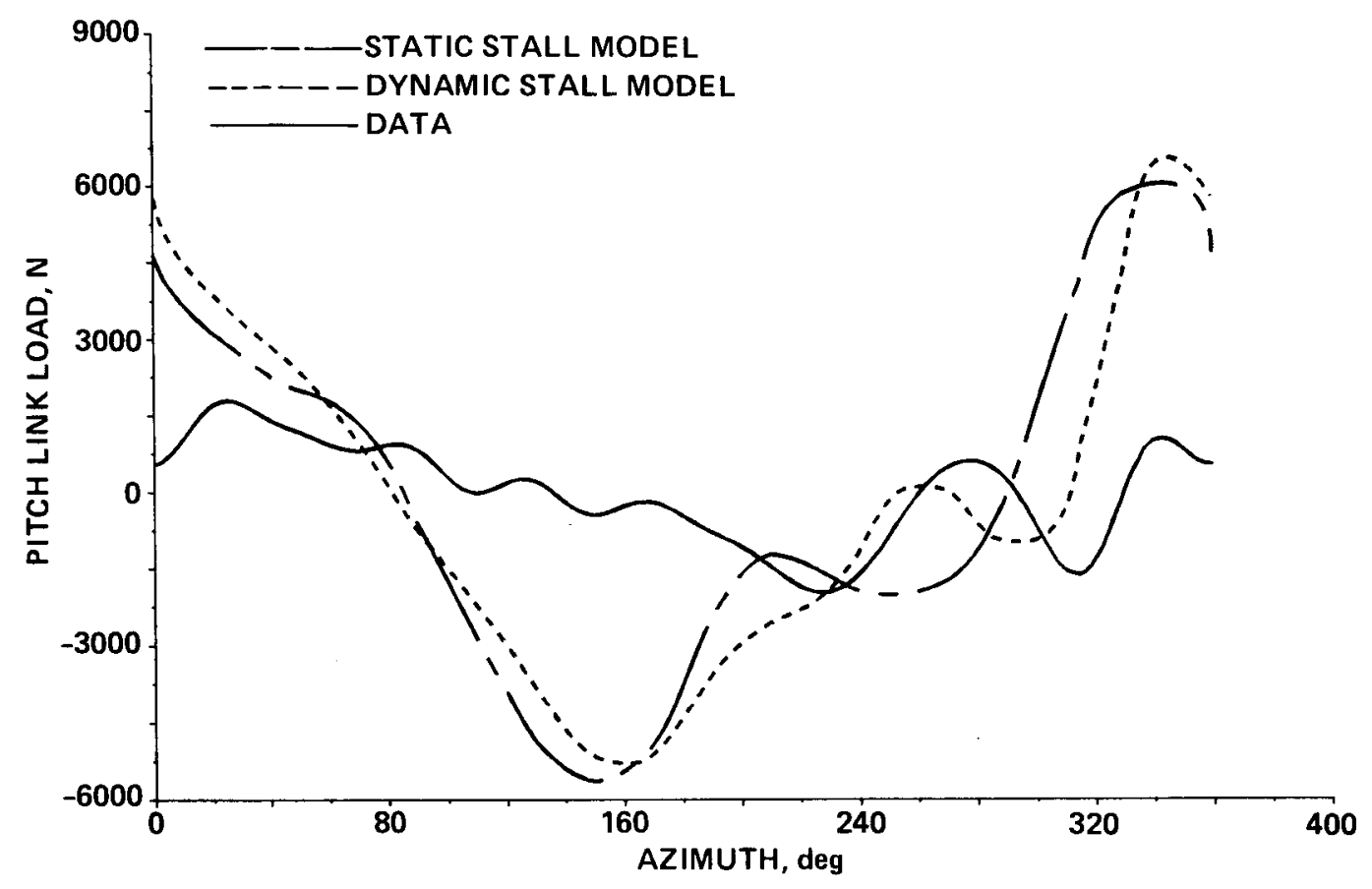

Fig. 30. Effect of stall model on pitch link loads - Condition 3. 


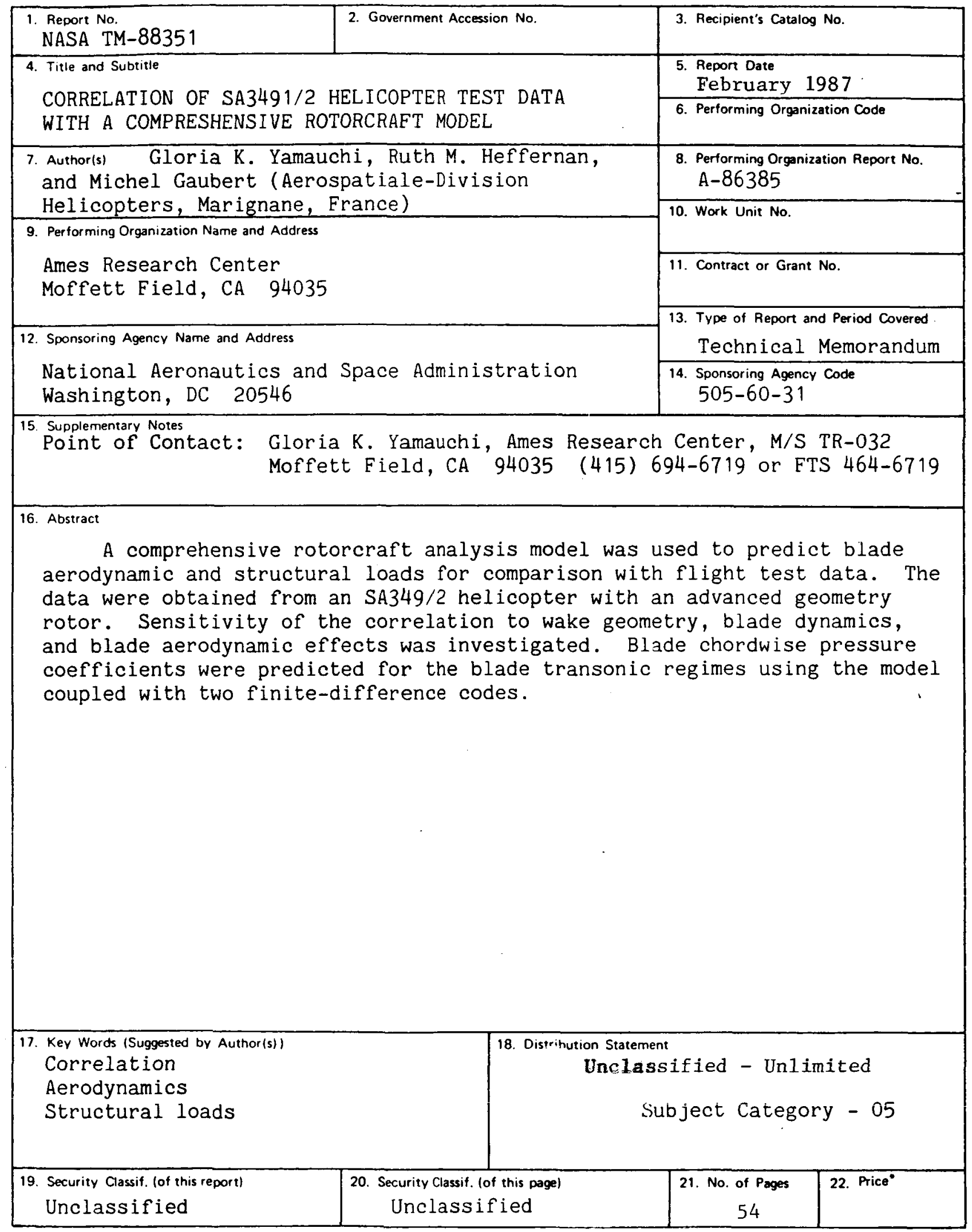

-For sale by the National Technical Information Service, Springfield, Virginia 22161 